\title{
Least-Squares Residual Power Series Method for the Time-Fractional Differential Equations
}

\author{
Jianke Zhang $\mathbb{D}^{1,}{ }^{1,2}$ Zhirou Wei, ${ }^{1}$ Lifeng $\mathrm{Li}^{1}{ }^{1}$ and Chang $\mathrm{Zhou}^{1}$ \\ ${ }^{1}$ School of Science, Xi'an University of Post and Telecommunications, Xi'an 710121, China \\ ${ }^{2}$ Shaanxi Key Laboratory of Network Data Analysis and Intelligent Processing, \\ Xi'an University of Posts and Telecommunications, Xi'an, Shaanxi 710121, China \\ Correspondence should be addressed to Jianke Zhang; jiankezh@163.com
}

Received 16 June 2019; Accepted 18 September 2019; Published 30 October 2019

Academic Editor: Ludovico Minati

Copyright () 2019 Jianke Zhang et al. This is an open access article distributed under the Creative Commons Attribution License, which permits unrestricted use, distribution, and reproduction in any medium, provided the original work is properly cited.

In this study, an applicable and effective method, which is based on a least-squares residual power series method (LSRPSM), is proposed to solve the time-fractional differential equations. The least-squares residual power series method combines the residual power series method with the least-squares method. These calculations depend on the sense of Caputo. Firstly, using the classic residual power series method, the analytical solution can be solved. Secondly, the concept of fractional Wronskian is introduced, which is applied to validate the linear independence of the functions. Thirdly, a linear combination of the first few terms as an approximate solution is used, which contains unknown coefficients. Finally, the least-squares method is proposed to obtain the unknown coefficients. The approximate solutions are solved by the least-squares residual power series method with the fewer expansion terms than the classic residual power series method. The examples are shown in datum and images. The examples show that the new method has an accelerate convergence than the classic residual power series method.

\section{Introduction}

The fractional calculation has been a popular topic for the past few years. Leibniz and L'Hopital were the first to propose fractional differential equations. Fractional differential equations are applied to many different fields, such as control science and engineering [1] and computer science and technology [2]. Many researchers have studied different theories in fractional differential equations. There are many different approximate analytical methods to solve fractional differential equations, such as the homotopy analysis transform method [3], (G//G)-expansion method [4], homotopy perturbation method [5], and variational iteration method [6].

In recent years, a new method has been proposed to solve the fractional differential equations by the fractional residual power series method, which was used to find the analytical solution for several classes of fractional differential equations. Many scholars have devoted themselves to the study of this field. In [7], the time-fractional foam drainage equation was worked out by the residual power series method. Wang and Chen [8] proposed that the residual power series can be applied to time-fractional Whitham-Broer-Kaup equations. The fractional variation of the $(1+1)$-dimensional BiswasMilovic equation [9] was figured out by the residual power series method. Alquran et al. [10] solved the time-fractional Phi-4 equation by the residual power method. A system of multipantograph delay differential equations using the residual power series method was calculated by Komashynska et al. [11]. The approximate solutions for the time-fractional Sharma-Tasso-Olever equation [12] were solved by the residual power series method. In [13], an approach was applied to find the exact solutions of fractional-order time-dependent Schrödinger equations. The residual power series method was also used for many other problems, such as the gas dynamic equation [14], the fractional initial Emden-Fowler equation [15], the generalized Berger-Fisher equation [16], and the nonlinear time-space-fractional Benney-Lin equation [17].

Recently, some existing methods have been modified by the least-squares method so that the approximate solution achieves higher accuracy. Kumar and Koundal [18] proposed an approach in which the system of nonlinear fractional partial differential equations was figured out by 
generalized least-squares homotopy perturbations. The approximate analytical solutions for nonlinear differential equations were solved by the least-squares homotopy perturbation method [19]. The linear and nonlinear fractional partial differential equations were figured out by the leastsquares homotopy perturbation method [20].

In this paper, the least-squares method is combined with the residual power series method, which is called the leastsquares residual power series method (LSRPSM). Compared with the classic residual power series method, a more accurate approximate solution with fewer expansion terms can be obtained by the new method.

The rest of the structure is as follows. In Section 2, some basic definitions about the sense of Caputo and fractional partial Wronskian are introduced. In Section 3, the leastsquares residual power series method is proposed with necessary definitions. Numerical results and discussions are presented by graphics and charts in Section 4. At last, the conclusion is drawn in Section 5.

\section{Basic Definitions}

In this section, the definition of the Caputo fractional is introduced systematically. This section also presents the definition of fractional partial Wronskian.

Definition 1 (see [21-23]). The Riemann-Liouville fractional integral operator of order $\alpha \geq 0$ is defined as

$$
J^{\alpha} f(t)= \begin{cases}\frac{1}{\Gamma(\alpha)} \int_{0}^{t} \frac{f(s)}{(t-s)^{1-\alpha}} \mathrm{d} s=\frac{1}{\Gamma(\alpha)} t^{\alpha-1} * f(t), & \alpha>0, t>0, \\ f(t), & \alpha=0,\end{cases}
$$

where $t^{\alpha-1} * f(t)$ is the convolution product of $t^{\alpha-1}$ and $f(t)$.

For the Riemann-Liouville fractional integral, we have

(1) $J^{\alpha} t^{\beta}=\Gamma(\beta+1) / \Gamma(\beta+\alpha+1) t^{\alpha+\beta}, \quad \beta>-1$,

(2) $J^{\alpha}(\lambda f(t)+\mu g(t))=\lambda J^{\alpha} f(t)+\mu J^{\alpha} g(t)$,

where $\lambda$ and $\mu$ are real constants.

Definition 2 (see $[24,25]$ ). Let $f(t):[0,+\infty)$ be a function and $\alpha$ be the upper positive integer of $\alpha(\alpha>0)$. The Caputo fractional derivative is defined by

$$
D^{\alpha} f(t)=\frac{1}{\Gamma(\alpha-\alpha)} \int_{0}^{t} \frac{f^{(\alpha)}(s)}{(t-s)^{\alpha+1-\alpha}} \mathrm{d} s, \quad \alpha-1<\alpha \leq \alpha, n \in N .
$$

For the Caputo derivative, we have

(1) $D^{\alpha} J^{\alpha} f(t)=f(t)$,

(2) $J^{\alpha} D^{\alpha} f(t)=f(t)-\sum_{i=0}^{n-1} y^{(i)}(0)\left(t^{i} / i !\right)$,

(3) $D^{\alpha} t^{\beta}= \begin{cases}(\Gamma(\beta+1) / \Gamma(\beta+1-\alpha)) t^{\beta-\alpha}, & \beta \geq \alpha, \\ 0, & \beta<\alpha,\end{cases}$

(4) $D^{\alpha} c=0$,

(5) $D^{\alpha}(\lambda f(t)+\mu g(t))=\lambda D^{\alpha} f(t)+\mu D^{\alpha} g(t)$,

where $\lambda, \mu$, and $c$ are real constants.

Definition 3 (see [18]). Let $\Phi_{1}, \Phi_{2}, \ldots, \Phi_{n}$ be $n$ functions of variables $x$ and $t$ which are defined on domain $\Omega$; then, fractional partial Wronskian of $\Phi_{1}, \Phi_{2}, \ldots, \Phi_{n}$ is

$$
W^{\alpha}\left[\Phi_{1}, \Phi_{2}, \ldots, \Phi_{n}\right]=\left|\begin{array}{ccccc}
\Phi_{1} & \Phi_{2} & \Phi_{3} & \cdots & \Phi_{n} \\
D^{\alpha} \Phi_{1} & D^{\alpha} \Phi_{2} & D^{\alpha} \Phi_{3} & \cdots & D^{\alpha} \Phi_{n} \\
D^{2 \alpha} \Phi_{1} & D^{2 \alpha} \Phi_{2} & D^{2 \alpha} \Phi_{3} & \cdots & D^{2 \alpha} \Phi_{n} \\
\vdots & \vdots & \vdots & \vdots & \vdots \\
D^{\alpha(n-1)} \Phi_{1} & D^{\alpha(n-1)} \Phi_{2} & D^{\alpha(n-1)} \Phi_{3} & \cdots & D^{\alpha(n-1)} \Phi_{n}
\end{array}\right|
$$

where $D^{\alpha}\left(\Phi_{i}\right)=\left(\partial / \partial x+\partial^{\alpha} / \partial t^{\alpha}\right)\left(\Phi_{i}\right)$ and $D^{n \alpha}=D^{\alpha}$. $D^{\alpha} \ldots D^{\alpha}(n-$ times $)$ for $0<\alpha \leq 1$ and $i=1,2,3, \ldots, n$.

Theorem 1 (see [18]). If the fractional partial Wronskian of $n$ functions $\Phi_{1}(x, t), \Phi_{2}(x, t), \ldots, \Phi_{n}(x, t)$ is nonzero, at last at one point of the domain $\Omega=[a, b] \times[a, b]$, then functions $\Phi_{1}(x, t), \Phi_{2}(x, t), \ldots, \Phi_{n}(x, t)$ are said to be linearly independent.

\section{Direct Method of Least-Squares Residual Power Series Method (LSRPSM)}

In this section, the general procedure of the least-squares residual power series method is proposed. Based on the classic residual power series method, the method of combining residual power series with the least-squares method is used for the time-fractional differential equations.

3.1. Classic Residual Power Series Method. We consider the following time-fractional differential equation:

$$
\mathscr{L}^{\alpha}(u(x, t))+\mathcal{N}(u(x, t))=0, \quad t>0,0<\alpha \leq 1,
$$

with

$$
I(u)=0
$$

where $\mathscr{L}$ is a linear operator, $\mathscr{N}$ is a nonlinear operator, $u(x, t)$ is an unknown function, and $I$ is an initial condition. 
Following the classic residual power series method $[26,27]$, the algorithm can be proposed by

$$
u(x, t)=\sum_{n=0}^{\infty} f_{n}(x) \frac{t^{n \alpha}}{\Gamma(1+n \alpha)} .
$$

In order to obtain the approximate value of (6), the form of the $i$ th series of $u(x, t)$ is proposed. Then, the truncated series $u_{i}(x, t)$ is defined by

$$
u_{i}(x, t)=\sum_{n=0}^{i} f_{n}(x) \frac{t^{n \alpha}}{\Gamma(1+n \alpha)} .
$$

With the initial condition $I(u)=0$, we define the $i$ th residual function as follows:

$$
\operatorname{Res}_{i}(x, t)=\mathscr{L}^{\alpha}\left(u_{i}(x, t)\right)+\mathscr{N}\left(u_{i}(x, t)\right) .
$$

In order to get $f_{n}(x), n \in N^{*}$, we look for the solution of

$$
D_{t}^{(n-1) \alpha} \operatorname{Res}_{i}(x, 0)=0, \quad i \in N^{*},
$$

where $N^{*}=\{1,2,3, \ldots\}$.

Here, classic residual power series method will give the $i^{\text {th }}$-order approximate solutions with

$$
u_{i}=\phi_{0}+\phi_{1}+u_{2}+\cdots+\phi_{i}
$$

where

$$
\begin{aligned}
& \phi_{0}=f_{0}(x), \\
& \phi_{1}=f_{1}(x) \frac{t^{\alpha}}{\Gamma(1+\alpha)}, \\
& \phi_{2}=f_{2}(x) \frac{t^{2 \alpha}}{\Gamma(1+2 \alpha)}, \\
& \vdots \\
& \phi_{i}=f_{i}(x) \frac{t^{i \alpha}}{\Gamma(1+i \alpha)} .
\end{aligned}
$$

Theorem 2 (see [28]) (convergence theorem). Suppose that $u(x, t) \in C\left(\left[r, t_{0}\right] \times\left[r, t_{0}+r\right]\right), D_{t}^{i \alpha} u(x, t) \in C\left(\left[r, t_{0}\right] \times\left[r, t_{0}+\right.\right.$ $r]$ ) for $i=0,1,2, \ldots, N+1$, where $0 \leq m-1<\alpha \leq m$ and $D_{t}^{i \alpha} u(x, t)$ can be differentiated $m-1$ with respect to $t$ on $\left(t_{0}, t_{0}+r\right)$. Then,

$$
u(x, t) \cong \sum_{j=0}^{m-1} \sum_{i=0}^{N} U_{j+i \alpha}(x)\left(t-t_{0}\right)^{j+i \alpha},
$$

where

$$
U_{j+i \alpha}(x)=\frac{D_{t}^{j+i \alpha}}{\Gamma(j+i \alpha+1)} u\left(x, t_{0}\right),
$$

and $r$ is the radius of convergence.

Moreover, there exists a value $\xi$, where $0 \leq \xi \leq t$ so that the error term $r_{N}(x, t)$ has the form

$$
\left\|r_{N}(x, t)\right\|=\operatorname{Sup}_{t \in[0, T]}\left|\sum_{j=0}^{m-1}\left(\frac{D^{(N+1) \alpha+j} u(x, \xi) t^{(N+1) \alpha+j}}{\Gamma((N+1) \alpha+i+1)}\right)\right| .
$$

According to Theorem 1, we can obtain

$$
\lim _{i \longrightarrow \infty} u_{i}(x, t)=u(x, t) \text {, }
$$

so

$$
\lim _{i \longrightarrow \infty} \operatorname{Res}\left(x, t, u_{i}(x, t)\right)=\operatorname{Res}(x, t, u(x, t))=0 .
$$

3.2. Least-Squares Residual Power Series Method. The procedure of the least-squares residual power series method is presented, and some definitions we require are proposed in this section.

Let the remainder $\widetilde{\text { Res }}$ for the differential equation (4) be

$$
\widetilde{\operatorname{Res}}(x, t, \widetilde{u})=\mathscr{L}^{\alpha}(\widetilde{u}(x, t))+\mathcal{N}(\widetilde{u}(x, t)), \quad x, t \in R,
$$

with the condition

$$
I(\widetilde{u})=0,
$$

where $\widetilde{u}$ is the approximate solution of equation (4).

Remark 1. If

$$
\lim _{i \longrightarrow \infty} \widetilde{\operatorname{Res}}\left(x, t, s_{i}^{\alpha}(x, t)\right)=0,
$$

$\left\{s_{i}^{\alpha}(x, t)\right\}_{i \in N^{*}}$ converge to the solution of equation (4).

Definition 4. We say $\tilde{u}$ is the $\varepsilon$-approximate residual power series method solution of equation (4) on domain $\Omega$ if

$$
|\widetilde{\operatorname{Res}}(x, t, \widetilde{u})|<\varepsilon,
$$

and (18) is also satisfied by $\widetilde{u}$.

Definition 5. We call $\tilde{u}$ is the weak $\varepsilon$-approximate residual power series method solution of equation (4) on domain $\Omega$ if

$$
\iint_{\Omega} \widetilde{\operatorname{Res}}^{2}(x, t, \widetilde{u}) \mathrm{d} x \mathrm{~d} t \leq \varepsilon,
$$

and (18) is also satisfied by $\widetilde{u}$.

We propose the following steps for the least-squares residual power series method.

Step 1. We use the classic residual power series method. The form of $u_{i}(x, t)$ can be written as

$$
u_{i}(x, t)=\sum_{n=0}^{i} f_{n}(x) \frac{t^{n \alpha}}{\Gamma(1+n \alpha)},
$$

and the $i$ th residual function is as follows:

$$
\operatorname{Res}_{i}(x, t)=\mathscr{L}^{\alpha}\left(u_{i}(x, t)\right)+\mathscr{N}\left(u_{i}(x, t)\right) .
$$

Then, we look for the solution of $f_{n}(x)$ by 


$$
D_{t}^{(n-1) \alpha} \operatorname{Res}_{i}(x, 0)=0, \quad i \in N^{*},
$$

where $N^{*}=\{1,2,3, \ldots\}$.

Here the classic residual power series method will give the $i^{\text {th }}$-order approximate solutions with

$$
W^{\alpha}\left[\phi_{0}, \phi_{1}, \phi_{2}, \ldots, \phi_{i}\right]=\mid \begin{gathered}
\phi_{0} \\
D^{\alpha} \phi_{0} \\
D^{2 \alpha} \phi_{0} \\
\vdots \\
D^{\alpha(n-1)} \phi_{0}
\end{gathered}
$$

where $D^{\alpha}=\left(\partial / \partial x+\partial^{\alpha} / \partial t^{\alpha}\right), 0<\alpha \leq 1$.

Let $S_{i}=\left\{\phi_{0}, \phi_{1}, \ldots, \phi_{i}\right\}$, where $i=0,1,2, \ldots$ and the elements of $S_{i}$ are linearly independent in the vector space of continuous functions defined on $R$.

Remark 2. If we cannot find the point so that the value of $W^{\alpha}\left[\phi_{0}, \phi_{1}, \phi_{2}, \ldots, \phi_{i}\right]$ is not 0 , the set $S_{i}$ is linearly dependent. So, we must use the classic residual power series method in this case.

Step 3. Assume

$$
\widetilde{u}_{i}=\sum_{n=0}^{i} k_{i}^{n} \phi_{r}
$$

as the approximate solution of equation (4). And substituting the approximate solution $\tilde{u}_{i}$ in (17), we obtain

$$
\widetilde{\operatorname{Res}}\left(x, t, k_{i}^{n}\right)=\widetilde{\operatorname{Res}}\left(x, t, \tilde{u}_{i}\right)
$$

Step 4. We attach to the following functional:

$$
\min J=\iint_{\Omega}\left(\widetilde{\operatorname{Res}}^{2}\left(x, t, k_{i}^{n}\right)\right) d x d t
$$

Here, we calculate some constants $k_{i}^{n}\left(k_{i}^{m+1}, k_{i}^{m+2}, \ldots, k_{i}^{n}\right)$.

We compute the values of $\tilde{k}_{i}^{m+1}, \widetilde{k}_{i}^{m+2}, \ldots, \tilde{k}_{i}^{n}$ as the values which give the minimum of (29) and the values of $\widetilde{k}_{i}^{0}, \widetilde{k}_{i}^{1}, \ldots, \widetilde{k}_{i}^{m}$ again as functions of $\widetilde{k}_{i}^{m+1}, \widetilde{k}_{i}^{m+2}, \ldots, \widetilde{k}_{i}^{n}$ by using the initial conditions.

So, we can obtain the value of $s_{i}^{\alpha}(x, t)$ by solving (29):

$$
s_{i}^{\alpha}(x, t)=\sum_{n=0}^{i} \widetilde{k}_{i}^{n} \phi_{r}
$$

From (27)-(30), we can get

$$
\widetilde{\operatorname{Res}}^{2}\left(x, t, s_{i}^{\alpha}(x, t)\right) \leq \widetilde{\operatorname{Res}}^{2}\left(x, t, \widetilde{u}_{i}(x, t)\right) .
$$

Theorem 3. The values of $s_{i}^{\alpha}(x, t)$ from (30) satisfy the property:

$$
u_{i}=\phi_{0}+\phi_{1}+\phi_{2}+\cdots+\phi_{i}
$$

where $\phi_{0}, \phi_{1}, \ldots, \phi_{i}$ can be calculated by (11).

Step 2. The linearly independent functions can be verified by

$$
\begin{array}{cccc}
\phi_{1} & \phi_{2} & \cdots & \phi_{i} \\
D^{\alpha} \phi_{1} & D^{\alpha} \phi_{2} & \cdots & D^{\alpha} \phi_{i} \\
D^{2 \alpha} \phi_{1} & D^{2 \alpha} \phi_{2} & \cdots & D^{2 \alpha} \phi_{i} \\
\vdots & \vdots & \vdots & \vdots \\
D^{\alpha(n-1)} \phi_{1} & D^{\alpha(n-1)} \phi_{2} & \cdots & D^{\alpha(n-1)} \phi_{i}
\end{array} \mid \neq 0,
$$

Proof. Based on the way that $s_{i}^{\alpha}(x, t)$ is computed, the following inequality holds:

$$
\iint_{\Omega} \widetilde{\operatorname{Res}}^{2}\left(x, t, s_{i}^{\alpha}(x, t)\right) \mathrm{d} x \mathrm{~d} t \geq 0 .
$$

Also, from (31) we have

$\iint_{\Omega} \widetilde{\operatorname{Res}}^{2}\left(x, t, u_{i}(x, t)\right) \mathrm{d} x \mathrm{~d} t \geq \iint_{\Omega} \widetilde{\operatorname{Res}}^{2}\left(x, t, s_{i}^{\alpha}(x, t)\right) \mathrm{d} x \mathrm{~d} t$, $\forall i \in N$.

Then, according to the convergence of the residual power series solution from (16), we can get

$$
\begin{aligned}
0 & \leq \lim _{i \rightarrow \infty} \iint_{\Omega} \widetilde{\operatorname{Res}}^{2}\left(x, t, s_{i}^{\alpha}(x, t)\right) \mathrm{d} x \mathrm{~d} t, \\
& \leq \lim _{i \rightarrow \infty} \iint_{\Omega} \widetilde{\operatorname{Res}}^{2}\left(x, t, u_{i}(x, t)\right) \mathrm{d} x \mathrm{~d} t=0 .
\end{aligned}
$$

The $\epsilon$-approximate residual power series solutions $s_{i}^{\alpha}(x, t)$ are also the weak solutions of equation (4).

\section{Illustrative Examples}

In this section, some examples are presented by the leastsquares residual power series method (LSRPSM). Using the new method, we usually compute the initial iterations by the fractional residual power series method, the rest can be ignored. Then, the least-squares method is applied, and the unknown coefficients are obtained. The approximate solutions are calculated by Maple in Windows 7 (64 bit). We analyse the approximate solutions by charts and graphics.

Example 1. Consider the following time-fractional Fornberg-Whitham equation:

$$
\begin{gathered}
D_{t}^{\alpha} u-D_{x x t} u+D_{x} u=u D_{x x x} u-u D_{x} u \\
+3 D_{x} u D_{x x} u, \quad t>0,0<\alpha \leq 1,
\end{gathered}
$$


with the initial condition

$$
u(x, 0)=\frac{4}{3} \exp \left(\frac{x}{2}\right)
$$

and the exact solution when $\alpha=1$ is

$$
u(x, t)=\frac{4}{3} \exp \left(\frac{x}{2}-\frac{2 t}{3}\right) .
$$

Using the classic residual power series method, we can obtain the following equations [29]:

$$
\begin{aligned}
& \phi_{0}(x, t)=\frac{4}{3} \exp \left(\frac{x}{2}\right) \\
& \phi_{1}(x, t)=-\frac{2}{3} \exp \left(\frac{x}{2}\right) \frac{t^{\alpha}}{\Gamma(1+\alpha)} \\
& \phi_{2}(x, t)=\frac{1}{3} \exp \left(\frac{x}{2}\right) \frac{t^{2 \alpha}}{\Gamma(1+2 \alpha)}
\end{aligned}
$$

Then,

$$
W^{\alpha}\left[\phi_{0}, \phi_{1}, \phi_{2}\right]=\left|\begin{array}{ccc}
\frac{4}{3} \exp \left(\frac{x}{2}\right) & -\frac{2}{3} \exp \left(\frac{x}{2}\right) \frac{t^{\alpha}}{\Gamma(1+\alpha)} & \frac{1}{3} \exp \left(\frac{x}{2}\right) \frac{t^{2 \alpha}}{\Gamma(1+2 \alpha)} \\
D^{\alpha} \frac{4}{3} \exp \left(\frac{x}{2}\right) & D^{\alpha}\left(-\frac{2}{3} \exp \left(\frac{x}{2}\right) \frac{t^{\alpha}}{\Gamma(1+\alpha)}\right) & D^{\alpha} \frac{1}{3} \exp \left(\frac{x}{2}\right) \frac{t^{2 \alpha}}{\Gamma(1+2 \alpha)} \\
D^{2 \alpha} \frac{4}{3} \exp \left(\frac{x}{2}\right) & D^{2 \alpha}\left(-\frac{2}{3} \exp \left(\frac{x}{2}\right) \frac{t^{\alpha}}{\Gamma(1+\alpha)}\right) & D^{2 \alpha} \frac{1}{3} \exp \left(\frac{x}{2}\right) \frac{t^{2 \alpha}}{\Gamma(1+2 \alpha)}
\end{array}\right| .
$$

When $\alpha=1, x=0, t=0.5, W^{1}\left[\phi_{0}, \phi_{1}, \phi_{2}\right] \neq 0$. Hence, the functions $\phi_{0}, \phi_{1}$, and $\phi_{2}$ are linearly independent.

So, we can obtain the approximate solution, which can be written as

$$
\begin{aligned}
\widetilde{u}= & k_{0} \frac{4}{3} \exp \left(\frac{x}{2}\right)+k_{1}\left(-\frac{2}{3} \exp \left(\frac{x}{2}\right) \frac{t^{\alpha}}{\Gamma(1+\alpha)}\right) \\
& +k_{2} \frac{1}{3} \exp \left(\frac{x}{2}\right) \frac{t^{2 \alpha}}{\Gamma(1+2 \alpha)} .
\end{aligned}
$$

From (36), we can get the residual function

$$
\begin{aligned}
\widetilde{\operatorname{Res}}(x, t, \widetilde{u})= & D_{t}^{\alpha} \widetilde{u}-D_{x x t} \widetilde{u}+D_{x} \widetilde{u}-\widetilde{u} D_{x x x} \widetilde{u}+\widetilde{u} D_{x} \widetilde{u} \\
& -3 D_{x} \widetilde{u} D_{x x} \widetilde{u},
\end{aligned}
$$

with the initial condition

$$
\widetilde{u}(x, 0)=\frac{4}{3} \exp \left(\frac{x}{2}\right) .
$$

Using the given condition (43) in (41), we get $k_{0}=1$. So, (41) can be written as

$$
\begin{aligned}
\widetilde{u}= & \frac{4}{3} \exp \left(\frac{x}{2}\right)+k_{1}\left(-\frac{2}{3} \exp \left(\frac{x}{2}\right) \frac{t^{\alpha}}{\Gamma(1+\alpha)}\right) \\
& +k_{2} \frac{1}{3} \exp \left(\frac{x}{2}\right) \frac{t^{2 \alpha}}{\Gamma(1+2 \alpha)} .
\end{aligned}
$$

So, we can obtain $\widetilde{R e s}$ by substituting (44) into (42). Then, the functional $J$ will be

$$
J\left(k_{1}, k_{2}\right)=\iint_{\Omega} \widetilde{\operatorname{Res}}^{2}\left(x, t, k_{1}, k_{2}\right) \mathrm{d} x \mathrm{~d} t .
$$

We compute the functional $J$ of (45). We receive two algebraic equations as

$$
\begin{gathered}
\frac{\partial J}{\partial k_{1}}=0, \\
\frac{\partial J}{\partial k_{2}}=0,
\end{gathered}
$$

and then we determine the unknown coefficients of (46) when $\alpha=1$ :

$$
\begin{aligned}
& k_{1}=1.29503916449089, \\
& k_{2}=1.28807658833778 .
\end{aligned}
$$

In Figure 1, the exact solutions and the approximate solutions using the least-squares residual power series method are presented by the three-dimensional graphics. Figure 1(a) presents the exact solution and Figure 1(b) presents the approximate solution when $\alpha=1,0 \leq t \leq 1,-10 \leq x \leq 10$. There are little differences between Figures 1(a) and 1(b). So, the approximate solution is accurate when the values of $\alpha$ approach 1 .

We present the absolute errors between the exact solutions and the approximate solutions by the new method. The absolute error can be written as

$$
\text { error }=\left|\widetilde{u}_{i}(x, t)-u(x, t)\right| \text {. }
$$

As is shown in Table 1, we present the absolute error between different values of $x$ and $t$ when $\alpha=1,0 \leq t \leq 1,-10 \leq x \leq 10$. For each item in the table, the upper corner is the name of the method, and the lower corner is the number of items expanded under the method. And the least-squares residual power series method (LSRPSM) with $\widetilde{u}_{i}(x, t)$ when $i=2$ is compared with the classic residual power series method (RPSM) with $u_{i}(x, t)$ when $i=5$, as shown in Table 1 .

From Table 1, we can find that the absolute errors by the least-squares residual power series method with different $x$ 


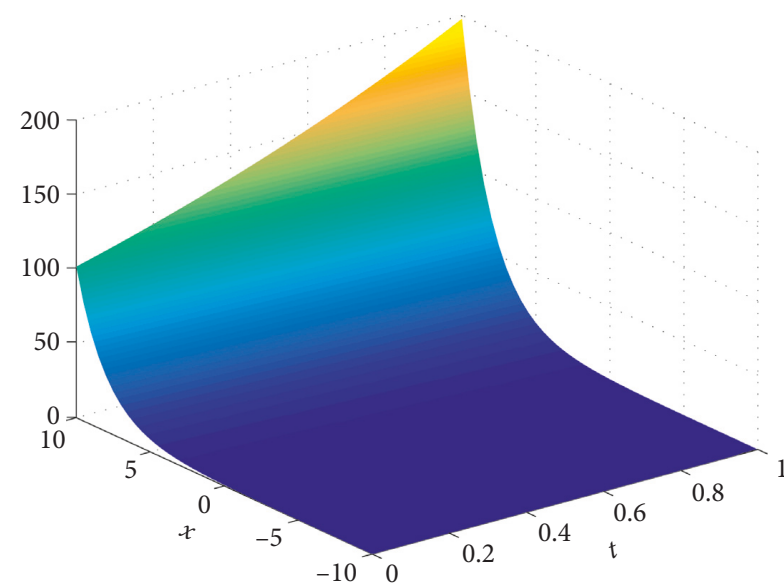

(a)

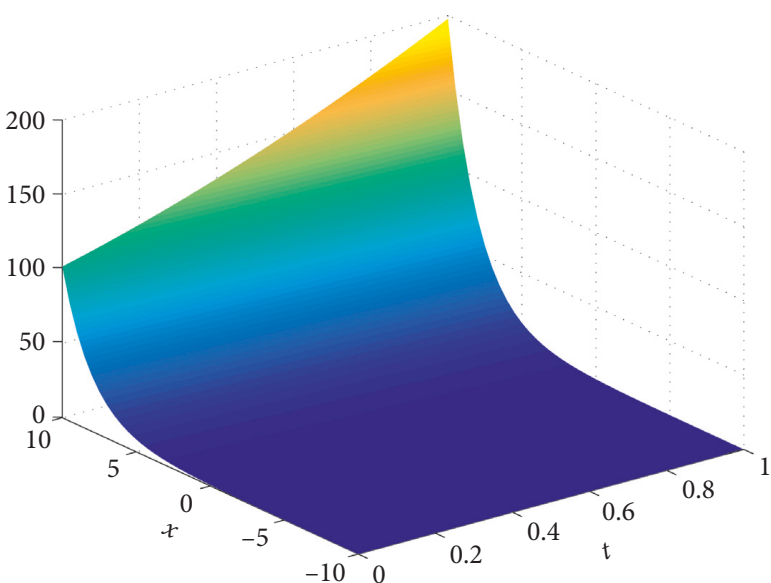

(b)

FIGURE 1: 3D graphics of exact and approximate solutions. (a) $\tilde{u}(x, t)_{\text {exact }}$. (b) $\tilde{u}(x, t)_{\text {LSRPSM }}$.

TABLE 1: Absolute errors by LSRPSM and RPSM for $\alpha=1$.

\begin{tabular}{cccccc}
\hline$x$ & $t$ & $u(x, t)_{\text {exact }}$ & $\widetilde{u}_{i}(x, t)_{i=2}^{\mathrm{LSRPS}}$ & Error $(x, t)_{i=2}^{\mathrm{LSRPS}}$ & Error $(x, t)_{i=5}^{\mathrm{RPSM}}$ \\
\hline & 0.1 & $8.4045 \times 10^{-3}$ & $8.4167 \times 10^{-3}$ & $1.2138659 \times 10^{-5}$ & $1.4124929 \times 10^{-4}$ \\
-10 & 0.2 & $7.8625 \times 10^{-3}$ & $7.8783 \times 10^{-3}$ & $1.5839983 \times 10^{-5}$ & $1.5839983 \times 10^{-5}$ \\
& 0.3 & $7.3554 \times 10^{-3}$ & $7.3689 \times 10^{-3}$ & $1.3513929 \times 10^{-5}$ & $3.7712027 \times 10^{-4}$ \\
& 0.4 & $6.8810 \times 10^{-3}$ & $6.8885 \times 10^{-3}$ & $7.4149830 \times 10^{-6}$ & $4.7437238 \times 10^{-4}$ \\
& 0.5 & $6.4373 \times 10^{-3}$ & $6.4369 \times 10^{-3}$ & $3.4775600 \times 10^{-7}$ & $5.5942160 \times 10^{-4}$ \\
\hline & 0.1 & $1.0239 \times 10^{-1}$ & $1.0254 \times 10^{-1}$ & $1.4787910 \times 10^{-4}$ & $1.7207686 \times 10^{-3}$ \\
& 0.2 & $9.5785 \times 10^{-2}$ & $9.5978 \times 10^{-2}$ & $1.9297050 \times 10^{-4}$ & $3.2466366 \times 10^{-3}$ \\
& 0.3 & $8.9607 \times 10^{-2}$ & $8.9772 \times 10^{-2}$ & $1.6463335 \times 10^{-4}$ & $4.5942654 \times 10^{-3}$ \\
& 0.4 & $8.3828 \times 10^{-2}$ & $8.3919 \times 10^{-2}$ & $9.0332970 \times 10^{-5}$ & $5.7790387 \times 10^{-3}$ \\
& 0.5 & $7.8422 \times 10^{-2}$ & $7.8418 \times 10^{-2}$ & $4.2365400 \times 10^{-6}$ & $6.8151503 \times 10^{-3}$ \\
\hline & 0.1 & 2.0565 & 2.0595 & $2.9702320 \times 10^{-3}$ & $3.4562561 \times 10^{-2}$ \\
& 0.2 & 1.9239 & 1.9278 & $3.8759170 \times 10^{-3}$ & $6.5210440 \times 10^{-2}$ \\
& 0.3 & 1.7998 & 1.8031 & $3.3067490 \times 10^{-3}$ & $9.2278286 \times 10^{-2}$ \\
& 0.4 & 1.6837 & 1.6856 & $1.8143870 \times 10^{-3}$ & $1.1607510 \times 10^{-1}$ \\
& 0.5 & 1.5751 & 1.5751 & $8.5092000 \times 10^{-5}$ & $1.3688595 \times 10^{-1}$ \\
\hline
\end{tabular}

and $t$ between the approximate solutions and the exact solutions are within the acceptable range. The range of magnitude of absolute errors is from $10^{-3}$ to $10^{-7}$. The smaller the value of the variable $t$ with fixed $x$ is, the bigger the absolute errors are. In the meantime, the smaller the value of the variable $x$ with fixed $t$ is, the smaller the absolute errors are. Comparing the classical method with our new method, we found that the new method has high accuracy.

Using the same method, the linearly independent functions can be verified when $\alpha=0.35,0.55,0.75,0.95$. Then, we can obtain the unknown coefficients $k_{1}$ and $k_{2}$, respectively, by using the least-squares method.

Figure 2 shows the influence of different $\alpha$ on the approximate solutions when $0 \leq t \leq 1,-10 \leq x \leq 10$. Figure $2(\mathrm{a})$ presents approximate solutions for $\alpha=0.35$, Figure 2(b) presents approximate solutions when $\alpha=0.55$, and Figures 2(c) and 2(d) show the approximate solutions when $\alpha=0.75$ and $\alpha=0.95$.

From Figure 2, the larger the value of $\alpha$, the smoother the plane. As the parameter $\alpha$ increases, the graphics get closer and closer to the exact solution of the graphic.
For any $\alpha \in(0,1]$, the exact value of $|\widetilde{\operatorname{Res}}(x, t)|$ is 0 . The distinction between the $2^{\text {th }}$ approximate solutions and the exact solutions can be shown by the values of $|\widetilde{\operatorname{Res}}(x, t)|$. So, we can use the values of $|\widetilde{\operatorname{Res}}(x, t)|$ to represent the deviation between the approximate solution and the exact solution.

Using the same method, the linearly independent functions can be verified when $\alpha=0.2,0.4$. Then, the unknown coefficients $k_{1}$ and $k_{2}$ can be solved, respectively, by using the least-squares method. And we compare the results of the least-squares residual power series method (LSRPSM), the residual power series method (RPSM) and the variational iteration method (VIM) [30]. The approximate solutions of VIM can be written as

$$
\begin{aligned}
& u_{0}(x, t)=\frac{4}{3} \exp \left(\frac{x}{2}\right) \\
& u_{1}(x, t)=\frac{2}{3} \exp \left(\frac{x}{2}\right)(2-t), \\
& u_{2}(x, t)=\frac{1}{6} \exp \left(\frac{x}{2}\right)\left(8-9 t+t^{2}+\frac{4 t^{2-\alpha}}{\Gamma(3-\alpha)}\right) .
\end{aligned}
$$




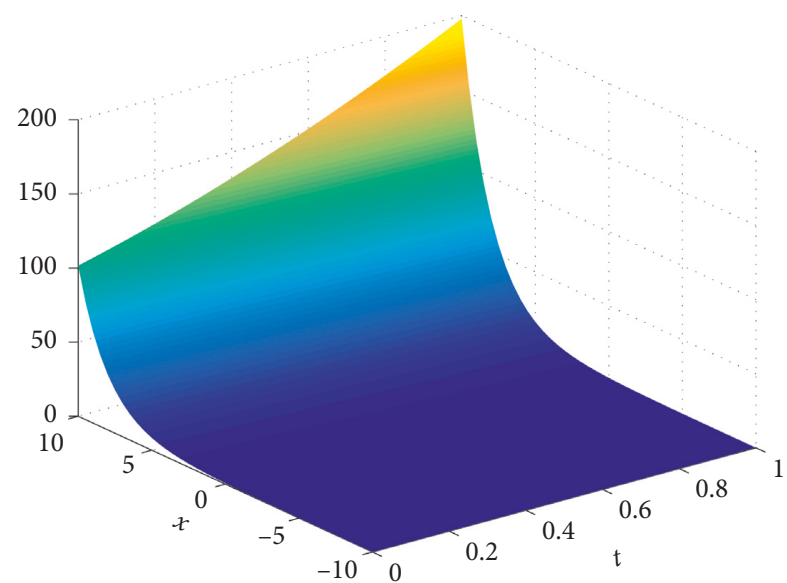

(a)

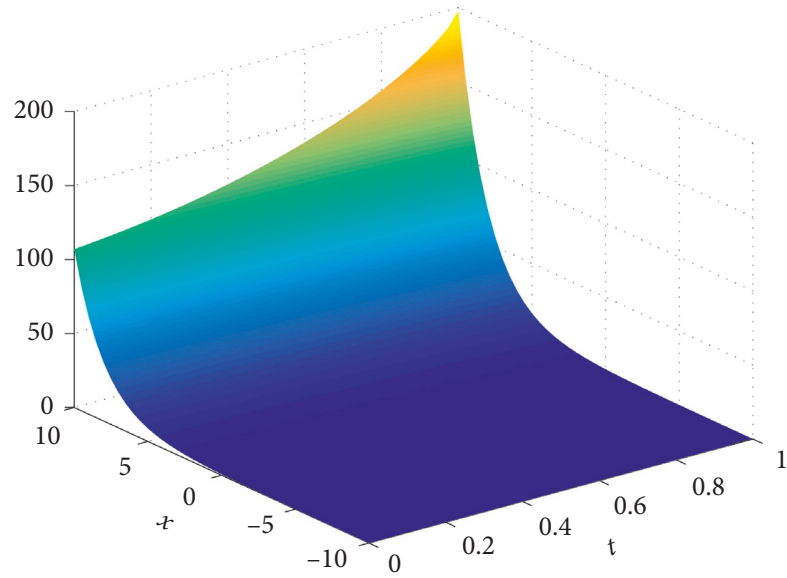

(c)

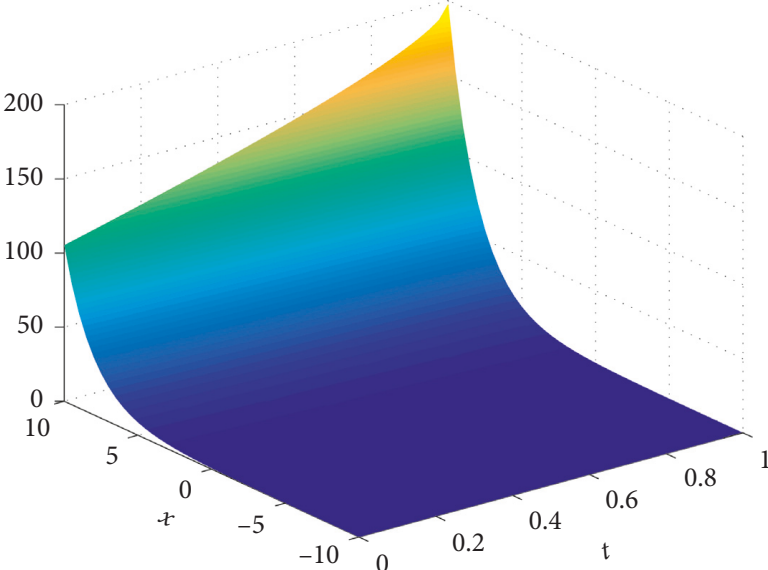

(b)

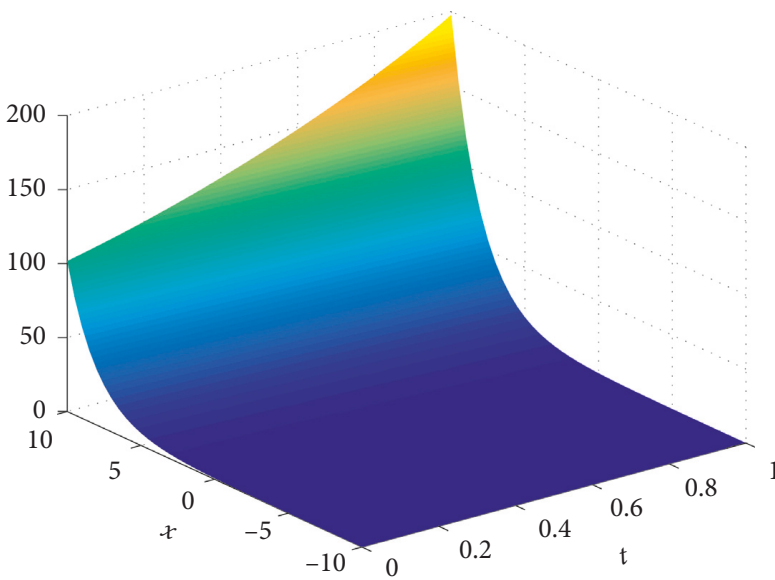

(d)

Figure 2: Approximate solution: (a) $\widetilde{u}(x, t, \alpha=0.35)$; (b) $\widetilde{u}(x, t, \alpha=0.55)$; (c) $\tilde{u}(x, t, \alpha=0.75)$; (d) $\tilde{u}(x, t, \alpha=0.95)$.

TABLE 2: The values of $|\widetilde{\operatorname{Res}}(x, t)|$ by LSRPSM, RPSM, and VIM for $\alpha=0.2,0.4$ and $x=-10$.

\begin{tabular}{|c|c|c|c|c|c|c|c|c|}
\hline \multirow[b]{2}{*}{$t$} & \multicolumn{4}{|c|}{$\alpha=0.2$} & \multicolumn{4}{|c|}{$\alpha=0.4$} \\
\hline & $\tilde{u}$ & $|\widetilde{\operatorname{Re} s}|_{i=2}^{\text {LSRPSM }}$ & $|\operatorname{Res}|_{i=2}^{\mathrm{RPSM}}$ & $|\operatorname{Res}|_{i=2}^{\mathrm{VIM}}$ & $\tilde{u}$ & $|\widetilde{\operatorname{Res}}|_{i=2}^{\text {LSRPSM }}$ & $|\operatorname{Res}|_{i=2}^{\mathrm{RPSM}}$ & $|\operatorname{Res}|_{i=2}^{\mathrm{VIM}}$ \\
\hline 0.1 & 0.00672 & 0.00066 & 0.00104 & 0.00465 & 0.00751 & 0.00194 & 0.00144 & 0.00361 \\
\hline 0.2 & 0.00660 & 0.00057 & 0.00089 & 0.00292 & 0.00704 & 0.00121 & 0.00100 & 0.00187 \\
\hline 0.3 & 0.00655 & 0.00063 & 0.00090 & 0.00144 & 0.00670 & 0.00082 & 0.00089 & 0.00054 \\
\hline 0.4 & 0.00653 & 0.00072 & 0.00095 & 0.00016 & 0.00642 & 0.00056 & 0.00088 & 0.00052 \\
\hline 0.5 & 0.00652 & 0.00080 & 0.00100 & 0.00096 & 0.00618 & 0.00036 & 0.00091 & 0.00139 \\
\hline
\end{tabular}

Table 2 presents the approximate solutions and the values of $|\widetilde{\operatorname{Res}}(x, t)|$ when $x=-10$.

Example 2. Consider the following time-fractional BBMBurger equation:

$$
D_{t}^{\alpha} u-u_{x x t}+u_{x}+\left(\frac{u^{2}}{2}\right)_{x}=0, \quad t>0,0<\alpha \leq 1,
$$

with the initial condition

$$
u(x, 0)=\operatorname{sech}^{2}\left(\frac{x}{4}\right)
$$

and the exact solution when $\alpha=1$ is

$$
u(x, t)=\operatorname{sech}^{2}\left(\frac{x}{4}-\frac{t}{4}\right)
$$

Using the classic residual power series method, we can obtain the following equations [31]: 


$$
\begin{aligned}
\phi_{0}(x, t)= & \operatorname{sech}^{2}\left(\frac{x}{4}\right) \\
\phi_{1}(x, t)= & \left(\frac{1}{2} \operatorname{sech}^{2}\left(\frac{x}{4}\right) \tanh \left(\frac{x}{4}\right)+\frac{1}{2} \operatorname{sech}\left(\frac{x}{4}\right) \tanh \left(\frac{x}{4}\right)\right) \frac{t^{\alpha}}{\Gamma(1+\alpha)} \\
\phi_{2}(x, t)= & \left(\frac{7}{8} \operatorname{sech}^{6}\left(\frac{x}{4}\right) \tanh ^{2}\left(\frac{x}{4}\right)-\frac{1}{8} \operatorname{sech}^{6}\left(\frac{x}{4}\right)\right. \\
& +\frac{5}{4} \operatorname{sech}^{4}\left(\frac{x}{4}\right) \tanh ^{2}\left(\frac{x}{4}\right)-\frac{1}{4} \operatorname{sech}^{4}\left(\frac{x}{4}\right) \\
& \left.+\frac{3}{8} \operatorname{sech}\left(\frac{x}{4}\right) \tanh ^{2}\left(\frac{x}{4}\right)-\frac{1}{8} \operatorname{sech}^{2}\left(\frac{x}{4}\right)\right) \frac{t^{2 \alpha}}{\Gamma(1+2 \alpha)}
\end{aligned}
$$

Then,

$$
W^{\alpha}\left[\phi_{0}, \phi_{1}, \phi_{2}\right]=\left|\begin{array}{ccc}
\phi_{0} & \phi_{1} & \phi_{2} \\
D^{\alpha} \phi_{0} & D^{\alpha} \phi_{1} & D^{\alpha} \phi_{2} \\
D^{2 \alpha} \phi_{0} & D^{2 \alpha} \phi_{1} & D^{2 \alpha} \phi_{2}
\end{array}\right| .
$$

When $\quad \alpha=1, x=2, t=1, \quad W^{1}\left[\phi_{0}, \phi_{1}, \phi_{2}\right]=$ $-0.2026562308 \neq 0$. Hence, the functions $\phi_{0}, \phi_{1}$, and $\phi_{2}$ are linearly independent.

So, we can obtain the approximate solution, which can be written as

$$
\begin{aligned}
\widetilde{u}= & k_{0} \cdot \operatorname{sech}^{2}\left(\frac{x}{4}\right)+k_{1} \cdot\left(\frac{1}{2} \operatorname{sech}^{2}\left(\frac{x}{4}\right) \tanh \left(\frac{x}{4}\right)\right. \\
& \left.+\frac{1}{2} \operatorname{sech}^{4}\left(\frac{x}{4}\right) \tanh \left(\frac{x}{4}\right)\right) \frac{t^{\alpha}}{\Gamma(1+\alpha)} \\
& +k_{2} \cdot\left(\frac{7}{8} \operatorname{sech}^{6}\left(\frac{x}{4}\right) \tanh ^{2}\left(\frac{x}{4}\right)-\frac{1}{8} \operatorname{sech}^{6}\left(\frac{x}{4}\right)\right. \\
& +\frac{5}{4} \operatorname{sech}^{4}\left(\frac{x}{4}\right) \tanh ^{2}\left(\frac{x}{4}\right)-\frac{1}{4} \operatorname{sech}^{4}\left(\frac{x}{4}\right)+\frac{3}{8} \operatorname{sech}^{2}\left(\frac{x}{4}\right) \tanh ^{2}\left(\frac{x}{4}\right) \\
& \left.-\frac{1}{8} \operatorname{sech}^{2}\left(\frac{x}{4}\right)\right) \frac{t^{2 \alpha}}{\Gamma(1+2 \alpha)} .
\end{aligned}
$$

From (50), we can get the residual function

$$
\widetilde{\operatorname{Res}}(x, t, \widetilde{u})=D_{t}^{\alpha} \widetilde{u}-\widetilde{u}_{x x t}+\widetilde{u}_{x}+\left(\frac{u^{2}}{2}\right)_{x},
$$

with the initial condition

$$
\widetilde{u}(x, 0)=\sec ^{2}\left(\frac{x}{4}\right) .
$$

Using the given condition (57) in (55), we get $k_{0}=1$. So, (55) can be written as

$$
\begin{aligned}
\tilde{u}= & \operatorname{sech}^{2}\left(\frac{x}{4}\right)+k_{1} \cdot\left(\frac{1}{2} \operatorname{sech}^{2}\left(\frac{x}{4}\right) \tanh \left(\frac{x}{4}\right)+\frac{1}{2} \operatorname{sech}^{4}\left(\frac{x}{4}\right)\right. \\
& \left.\cdot \tanh \left(\frac{x}{4}\right)\right) \frac{t^{\alpha}}{\Gamma(1+\alpha)}+k_{2} \cdot\left(\frac{7}{8} \operatorname{sech}^{6}\left(\frac{x}{4}\right) \tanh ^{2}\left(\frac{x}{4}\right)\right. \\
& -\frac{1}{8} \operatorname{sech}^{6}\left(\frac{x}{4}\right)+\frac{5}{4} \operatorname{sech}^{4}\left(\frac{x}{4}\right) \tanh ^{2}\left(\frac{x}{4}\right)-\frac{1}{4} \operatorname{sech}^{4}\left(\frac{x}{4}\right) \\
& \left.+\frac{3}{8} \operatorname{sech}^{2}\left(\frac{x}{4}\right) \tanh ^{2}\left(\frac{x}{4}\right)-\frac{1}{8} \operatorname{sech}^{2}\left(\frac{x}{4}\right)\right) \frac{t^{2 \alpha}}{\Gamma(1+2 \alpha)}
\end{aligned}
$$

So, we can obtain $\widetilde{\mathrm{Res}}$ by substituting (58) into (56). Then, the functional $J$ will be

$$
J\left(k_{1}, k_{2}\right)=\iint_{\Omega} \widetilde{\operatorname{Res}}^{2}\left(x, t, k_{1}, k_{2}\right) \mathrm{d} x \mathrm{~d} t
$$

We compute the functional $J$ of (59). We receive two algebraic equations as

$$
\begin{gathered}
\frac{\partial J}{\partial k_{1}}=0, \\
\frac{\partial J}{\partial k_{2}}=0,
\end{gathered}
$$

and then we determine the unknown coefficients of (60) when $\alpha=1$ :

$$
\begin{aligned}
& k_{1}=0.696922329601929, \\
& k_{2}=0.328042749015346 .
\end{aligned}
$$

In Figure 3, we present the exact solutions and the approximate solutions by the three-dimensional graphics. Figure 3(a) presents the exact solution and Figure 3(b) presents the approximate solution when $\alpha=1,0 \leq t \leq 1,-10 \leq x \leq 10$. From Figure 3, we can find that Figure $3(\mathrm{~b})$ is similar to Figure 3(a). So, the approximate solution is accurate when the values of $\alpha$ approach 1 .

As is shown in Table 3, we present the absolute error in (48) between different values of $x$ and $t$ when $\alpha=1,0 \leq t \leq 1,-2 \leq x \leq 2$. And the least-squares residual power series method (LSRPSM) and the q-homotopy analysis method (q-HAM) [32] with $\widetilde{u}_{i}(x, t)$ when $i=2$ are compared with the classic residual power series method with $u_{i}(x, t)$ when $i=2$, as shown in Table 3 . The approximate solutions of the q-HAM can be written as 


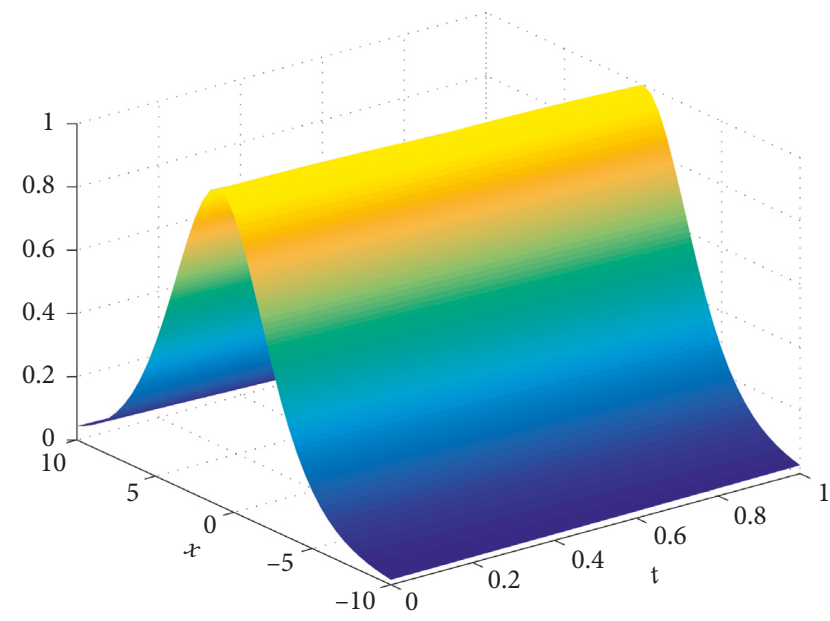

(a)

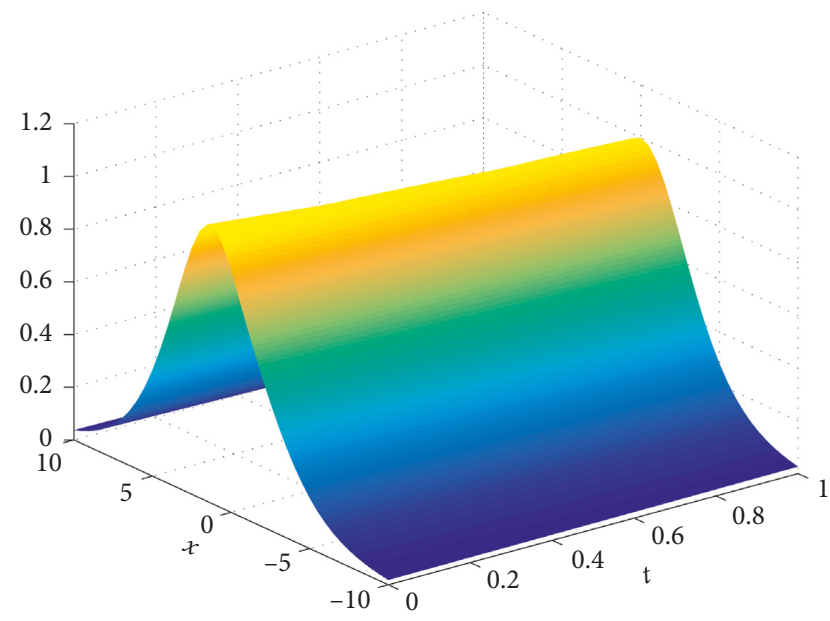

(b)

Figure 3: 3D graphics of exact and approximate solutions. (a) $\tilde{u}(x, t)_{\text {exact }}$ (b) $\tilde{u}(x, t)_{\text {LSRPSM }}$.

$$
\begin{aligned}
u_{2}(x, t ; n ; h)= & \operatorname{sech}\left(\frac{x}{4}\right)+\left(-\left(\frac{\varepsilon h}{8}\left(\cosh \left(\frac{x}{2}\right)-2\right)\right.\right. \\
& \left.+\frac{1}{2} \tanh \left(\frac{x}{2}\right)\right) \operatorname{sech}^{4}\left(\frac{x}{4}\right) \frac{t^{\alpha}}{\Gamma(\alpha+1)} \\
& \left.-\frac{1}{2} \tanh \left(\frac{x}{2}\right) \operatorname{sech}\left(\frac{x}{4}\right) \frac{t^{\alpha}}{\Gamma(\alpha+1)}\right)\left(\frac{1}{n}\right) \\
& +\left(( n + h ) \left(-\left(\frac{\varepsilon h}{8}\left(\cosh \left(\frac{x}{2}\right)-2\right)\right.\right.\right. \\
& +\frac{1}{2} \tanh \left(\frac{x}{2}\right) \operatorname{sech}^{4}\left(\frac{x}{4}\right) \frac{t^{\alpha}}{\Gamma(\alpha+1)} \\
& \left.-\frac{1}{2} \tanh \left(\frac{x}{2}\right)\right) \sec ^{2}\left(\frac{x}{4}\right) \frac{t^{\alpha}}{\Gamma(\alpha+1)}+\frac{h}{256} K \\
& \cdot(x, h, \varepsilon) \sec h^{7}\left(\frac{x}{4}\right) \frac{t^{2 \alpha-1}}{\Gamma(2 \alpha)}-\frac{h}{512} F(x, h, \varepsilon) \sec ^{8} \\
& \left.\left(\frac{x}{4}\right) \frac{t^{2 \alpha}}{\Gamma(2 \alpha+1)}\right)\left(\frac{1}{n}\right)^{2},
\end{aligned}
$$

where

$$
\begin{aligned}
K(x, h, \varepsilon)= & 40 \operatorname{ch} \cosh \left(\frac{x}{4}\right)-25 \operatorname{ch} \cosh \left(\frac{3 x}{4}\right)+\operatorname{ch} \cosh \left(\frac{5 x}{4}\right) \\
& -228 \sinh \left(\frac{x}{4}\right)+14 \sinh \left(\frac{3 x}{4}\right)+2 \sinh \left(\frac{5 x}{4}\right), \\
F(x, h, \varepsilon)= & 150 \operatorname{ch} \sinh \left(\frac{x}{2}\right)-2 \operatorname{sh} \sinh \left(\frac{3 x}{2}\right)-15 \operatorname{ch} \cosh \left(\frac{x}{2}\right) \\
& +24 \operatorname{chc} \cosh (x)-\varepsilon h \cosh \left(\frac{3 x}{2}\right)-40 \operatorname{ch}+214 \sinh \left(\frac{x}{2}\right) \\
& -16 \sinh (x)-2 \sinh \left(\frac{3 x}{2}\right)-92 \cosh \left(\frac{x}{2}\right) \\
& -64 \cosh (x)-4 \cosh \left(\frac{3 x}{2}\right)+416,
\end{aligned}
$$

and the values of parameters are $h=-1.5, \varepsilon=0$, and $n=1$.

From Table 3, we can obtain that the range of magnitude of absolute errors is from $10^{-3}$ to $10^{-8}$. The absolute errors by the new method with different values of $x$ and $t$ are within the acceptable range. Fix the value of $t$, and the absolute error is the smallest when $x=0$ and the absolute increases as the absolute value of $\mathrm{x}$ increases. Compared with the classic residual power series method and the q-homotopy analysis method, the leastsquares residual power series method is more accurate.

Using the same method, the linearly independent functions can be verified when $\alpha=0.3,0.6,0.9$. Then, we can obtain the unknown coefficients $k_{1}$ and $k_{2}$, respectively, by using the least-squares method.

Figure 4 shows the influence of different $\alpha$ on the approximate solutions when $0 \leq t \leq 1,-10 \leq x \leq 10$. Figures 4 (a)4 (c) present approximate solutions for $\alpha=0.3, \alpha=0.6$, and $\alpha=0.9$, respectively.

From Figure 4, we can conclude that the larger the value of $\alpha$, the smoother the images, and the closer to the image of the exact solution.

Using the same method, the linearly independent functions can be verified when $\alpha=0.7,0.9$. The unknown coefficients $k_{1}$ and $k_{2}$ can be obtained by using the leastsquares method. Table 4 shows the approximate solutions and the values of $|\widetilde{\operatorname{Res}}(x, t)|$ when $t=0.01$.

Example 3. Consider the following fractional biological population equation:

$$
\begin{array}{r}
D_{t}^{\alpha} u(x, y, t)=\left(u^{2}(x, y, t)\right)_{x x}+\left(u^{2}(x, y, t)\right)_{y y}+h u(x, y, t), \\
t>0,0<\alpha \leq 1,
\end{array}
$$

with the initial condition

$$
u(x, y, 0)=\sqrt{x y},
$$

and the exact solution when $\alpha=1$ is 
TABLE 3: Absolute errors by LSRPSM, RPSM, and q-HAM for $\alpha=1$.

\begin{tabular}{|c|c|c|c|c|c|c|}
\hline$x$ & $t$ & $u(x, t)_{\text {exact }}$ & $\widetilde{u}_{i}(x, t)_{i=2}^{\text {LSRPSM }}$ & Error $(x, t)_{i=2}^{\text {LSRPSM }}$ & $\operatorname{Error}(x, t)_{i=2}^{\text {RPSM }}$ & $\operatorname{Error}(x, t)_{i=2}^{\mathrm{q}-\mathrm{HAM}}$ \\
\hline \multirow{3}{*}{-2} & 0.001 & 0.7862659997 & 0.7862214953 & $4.45 \times 10^{-5}$ & $1.43 \times 10^{-4}$ & $2.55 \times 10^{-4}$ \\
\hline & 0.01 & 0.7846288220 & 0.7841854331 & $4.43 \times 10^{-3}$ & $1.43 \times 10^{-3}$ & $2.53 \times 10^{-3}$ \\
\hline & 0.1 & 0.7681097917 & 0.7638324718 & $4.28 \times 10^{-3}$ & $1.41 \times 10^{-2}$ & $2.36 \times 10^{-2}$ \\
\hline \multirow{3}{*}{-1} & 0.001 & 0.9398996870 & 0.9398591595 & $4.05 \times 10^{-5}$ & $1.08 \times 10^{-4}$ & $1.24 \times 10^{-4}$ \\
\hline & 0.01 & 0.9388589039 & 0.9384533623 & $4.06 \times 10^{-4}$ & $1.09 \times 10^{-3}$ & $1.27 \times 10^{-3}$ \\
\hline & 0.1 & 0.9280305730 & 0.9239405363 & $4.09 \times 10^{-3}$ & $1.19 \times 10^{-2}$ & $1.55 \times 10^{-2}$ \\
\hline \multirow{3}{*}{0} & 0.001 & 0.9999999376 & 0.9999999180 & $1.96 \times 10^{-8}$ & $1.88 \times 10^{-7}$ & $8.12 \times 10^{-7}$ \\
\hline & 0.01 & 0.9999937500 & 0.9999917989 & $1.95 \times 10^{-6}$ & $1.88 \times 10^{-5}$ & $8.13 \times 10^{-5}$ \\
\hline & 0.1 & 0.9993752604 & 0.9991798931 & $1.95 \times 10^{-4}$ & $1.88 \times 10^{-3}$ & $8.12 \times 10^{-3}$ \\
\hline \multirow{3}{*}{1} & 0.001 & 0.9401299142 & 0.9401704358 & $4.05 \times 10^{-5}$ & $1.08 \times 10^{-4}$ & $1.23 \times 10^{-4}$ \\
\hline & 0.01 & 0.9411611582 & 0.9415661251 & $4.05 \times 10^{-4}$ & $1.07 \times 10^{-3}$ & $1.16 \times 10^{-3}$ \\
\hline & 0.1 & 0.9510358396 & 0.9550681642 & $4.03 \times 10^{-3}$ & $9.76 \times 10^{-3}$ & $5.16 \times 10^{-3}$ \\
\hline \multirow{3}{*}{2} & 0.001 & 0.7866294307 & 0.7866739725 & $4.45 \times 10^{-5}$ & $1.43 \times 10^{-4}$ & $2.55 \times 10^{-4}$ \\
\hline & 0.01 & 0.7882631114 & 0.7887102046 & $4.47 \times 10^{-4}$ & $1.43 \times 10^{-3}$ & $2.53 \times 10^{-3}$ \\
\hline & 0.1 & 0.8044323122 & 0.8090801868 & $4.65 \times 10^{-3}$ & $1.45 \times 10^{-2}$ & $2.34 \times 10^{-2}$ \\
\hline
\end{tabular}

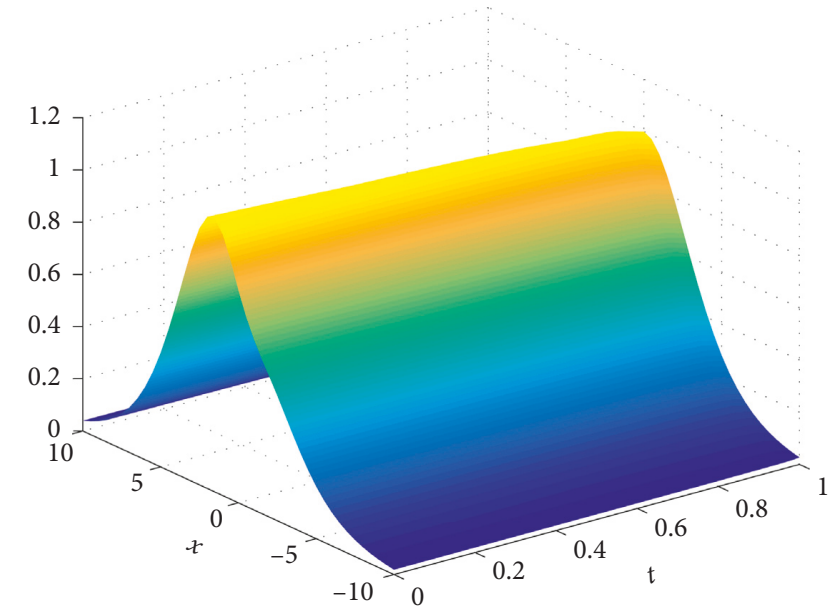

(a)

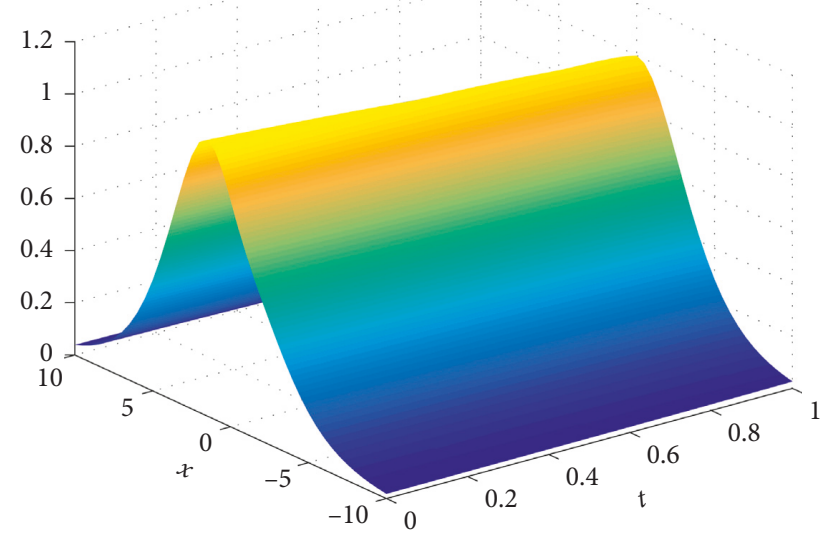

(b)

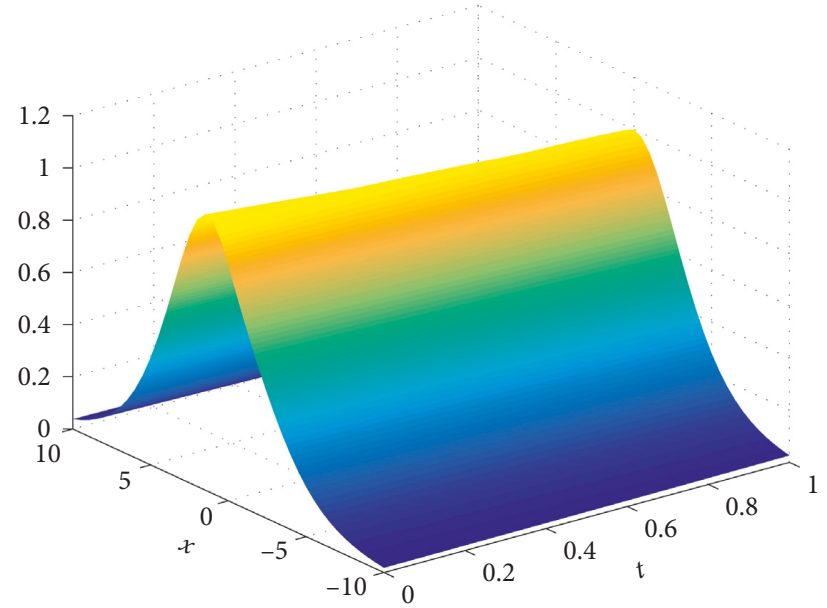

(c)

Figure 4: Approximate solution: (a) $\widetilde{u}(x, t, \alpha=0.3)$; (b) $\widetilde{u}(x, t, \alpha=0.6)$; (c) $\widetilde{u}(x, t, \alpha=0.9)$. 
TABLE 4: The values of $|\widetilde{\operatorname{Res}}(x, t)|$ by LSRPSM, RPSM, and q-HAM for $\alpha=0.7,0.9$ and $t=0.01$.

\begin{tabular}{|c|c|c|c|c|c|c|c|c|}
\hline \multirow[b]{2}{*}{$x$} & \multicolumn{4}{|c|}{$\alpha=0.7$} & \multicolumn{4}{|c|}{$\alpha=0.9$} \\
\hline & $\widetilde{u}$ & $|\widetilde{\operatorname{Res}}|_{i=2}^{\text {LSRPSM }}$ & $|\operatorname{Res}|_{i=2}^{\mathrm{RPSM}}$ & $|\operatorname{Res}|_{i=2}^{q-\mathrm{HAM}}$ & $\widetilde{u}$ & $|\widetilde{\operatorname{Res}}|_{i=2}^{\text {LSRPSM }}$ & $|\operatorname{Res}|_{i=2}^{\mathrm{RPSM}}$ & $|\operatorname{Res}|_{i=2}^{q-\mathrm{HAM}}$ \\
\hline 0 & 0.99983 & 0.01260 & 0.07816 & 0.28128 & 0.99998 & 0.00051 & 0.01180 & 0.05991 \\
\hline 0.5 & 0.98773 & 0.10168 & 0.18716 & 0.88821 & 0.98587 & 0.04172 & 0.11075 & 0.01272 \\
\hline 1 & 0.94605 & 0.17595 & 0.39198 & 0.98303 & 0.94249 & 0.06384 & 0.19674 & 0.10785 \\
\hline
\end{tabular}

$$
u(x, y, t)=\sqrt{x y} e^{h t} .
$$

Using the classic residual power series method, we can obtain the following equations [33]:

$$
\begin{aligned}
& \phi_{0}(x, t)=\sqrt{x y}, \\
& \phi_{1}(x, t)=h \sqrt{x y}, \\
& \phi_{2}(x, t)=h^{2} \sqrt{x y} .
\end{aligned}
$$

Then,

$$
W^{\alpha}\left[\phi_{0}, \phi_{1}, \phi_{2}\right]=\left|\begin{array}{ccc}
\sqrt{x y} & h \sqrt{x y} & h^{2} \sqrt{x y} \\
D^{\alpha} \sqrt{x y} & D^{\alpha}(h \sqrt{x y}) & D^{\alpha}\left(h^{2} \sqrt{x y}\right) \\
D^{2 \alpha} \sqrt{x y} & D^{2 \alpha}(h \sqrt{x y}) & D^{2 \alpha}\left(h^{2} \sqrt{x y}\right)
\end{array}\right| .
$$

When $\alpha=1, x=0.1, y=0.1, t=0.4, h=0.5, W^{1}\left[\phi_{0}, \phi_{1}\right.$, $\left.\phi_{2}\right] \neq 0$. Hence, the functions $\phi_{0}, \phi_{1}$, and $\phi_{2}$ are linearly independent.

So, we can obtain the approximate solution, which can be written as

$$
\widetilde{u}=k_{0} \cdot \sqrt{x y}+k_{1} \cdot h \sqrt{x y}+k_{2} \cdot h^{2} \sqrt{x y} .
$$

From (64), we can obtain the residual function

$$
\begin{aligned}
\widetilde{\operatorname{Res}}(x, t, \widetilde{u})= & D_{t}^{\alpha} \widetilde{u}(x, y, t)-\left(\widetilde{u}^{2}(x, y, t)\right)_{x x}-\left(\widetilde{u}^{2}(x, y, t)\right)_{y y} \\
& -h \widetilde{u}(x, y, t),
\end{aligned}
$$

with the initial condition

$$
\widetilde{u}(x, y, 0)=\sqrt{x y} .
$$

Using the given condition (71) in (69), we get $k_{0}=1$. So, (69) can be written as

$$
\widetilde{u}=\sqrt{x y}+k_{1} \cdot h \sqrt{x y}+k_{2} \cdot h^{2} \sqrt{x y} .
$$

So, we can obtain $\widetilde{R e s}$ by substituting (72) into (70). Then, the functional $J$ will be

$$
J\left(k_{1}, k_{2}\right)=\iiint_{\Omega} \widetilde{\operatorname{Res}}^{2}\left(x, y, t, k_{1}, k_{2}\right) \mathrm{d} x \mathrm{~d} y \mathrm{~d} t .
$$

We compute the functional $J$ of (73). We receive two algebraic equations as

$$
\begin{gathered}
\frac{\partial J}{\partial k_{1}}=0, \\
\frac{\partial J}{\partial k_{2}}=0,
\end{gathered}
$$

and then we determine the unknown coefficients of (74) when $\alpha=1$ :

$$
\begin{aligned}
& k_{1}=1.00001331097321, \\
& k_{2}=1.12959019129015 .
\end{aligned}
$$

From Figure 5, we obtain the exact solutions and the approximate solutions by the three-dimensional graphics. Figure 5(a) presents the exact solution, and Figure 5(b) presents the approximate solution. The condition of Figure 5 is $\alpha=1, t=1, h=0.5,-1 \leq x \leq 1,-1 \leq y \leq 1$. From Figure 5, we can conclude the three-dimensional graphics of the approximate solutions are similar to the exact solutions.

As is shown in Table 5, we present the absolute error in (49) between different values of $x$ and $t$ when $\alpha=1, t=1,0 \leq x \leq 1,0 \leq y \leq 1$. And the least-squares residual power series method (LSRPSM) with $\widetilde{u}_{i}(x, t)$ when $i=2$ is compared with the classic residual power series method and the homotopy perturbation method (HPM) [34] with $u_{i}(x, t)$ when $i=2$, as shown in Table 5 . The approximate solutions of HPM for $i=2$ can be written as

$$
u_{2}(x, t)=\sqrt{x y}+\frac{h t^{\alpha}}{\Gamma(1+\alpha)} \sqrt{x y}+\frac{h^{2} t^{2 \alpha}}{\Gamma(1+2 \alpha)} \sqrt{x y} .
$$

From Table 5, we can conclude that the absolute errors with the different values of $x$ and $y$ are within the acceptable range. Given the same items, we compare the residual power series method and the homotopy perturbation method with the least-squares residual power series method, and the new method is more accurate.

Using the least-squares residual power series method, the linearly independent functions can be verified when $\alpha=0.3,0.5,0.7,0.9$. Then, we can obtain the unknown 


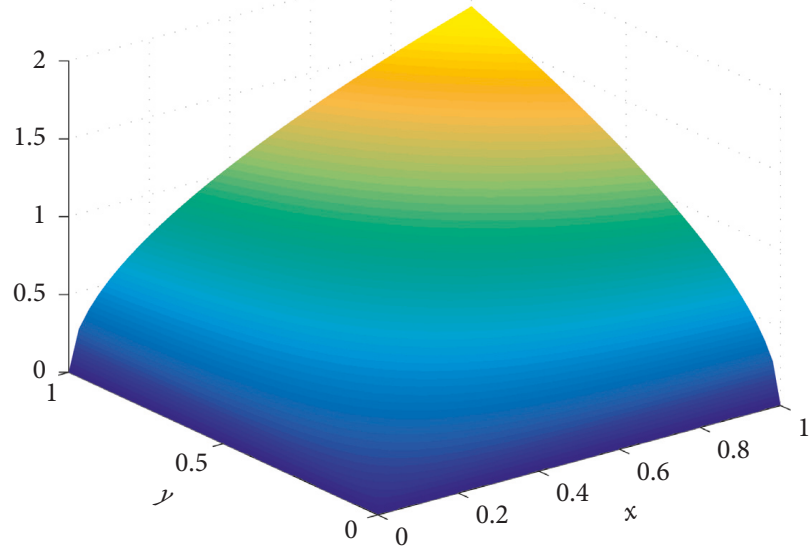

(a)

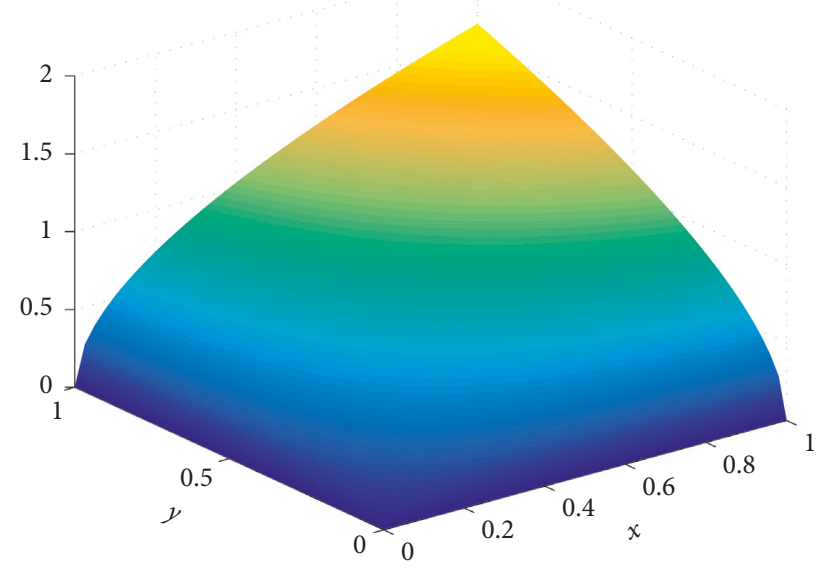

(b)

FIGURE 5: 3D graphics of exact and approximate solutions. (a) $\widetilde{u}(x, t)_{\text {exact }}$. (b) $\widetilde{u}(x, t)_{\text {LSRPSM }}$.

TABLE 5: Absolute errors by LSRPSM, RPSM, and HPM for $\alpha=1$.

\begin{tabular}{|c|c|c|c|c|c|c|}
\hline$x$ & $y$ & $u(x, t)_{\text {exact }}$ & $\tilde{u}_{i}(x, t)_{i=2}^{\text {LSRPM }}$ & Error $(x, t)_{i=2}^{\mathrm{RPSM}}$ & Error $(x, t)_{i=2}^{\text {LSRPSM }}$ & Error $(x, t)_{i=2}^{\mathrm{HPM}}$ \\
\hline \multirow{5}{*}{0.1} & 0.1 & 0.1648721271 & 0.1648625055 & $2.37 \times 10^{-3}$ & $9.62 \times 10^{-6}$ & $2.37 \times 10^{-3}$ \\
\hline & 0.2 & 0.2331643981 & 0.2331507911 & $3.35 \times 10^{-3}$ & $1.36 \times 10^{-5}$ & $3.35 \times 10^{-3}$ \\
\hline & 0.3 & 0.2855669010 & 0.2855502359 & $4.11 \times 10^{-3}$ & $1.67 \times 10^{-5}$ & $4.11 \times 10^{-3}$ \\
\hline & 0.4 & 0.3297442542 & 0.3297250110 & $4.74 \times 10^{-3}$ & $1.92 \times 10^{-5}$ & $4.74 \times 10^{-3}$ \\
\hline & 0.5 & 0.3686652837 & 0.3686437692 & $5.30 \times 10^{-3}$ & $2.15 \times 10^{-5}$ & $5.30 \times 10^{-3}$ \\
\hline \multirow{5}{*}{0.3} & 0.1 & 0.2855669010 & 0.2855502359 & $4.11 \times 10^{-3}$ & $1.67 \times 10^{-5}$ & $4.11 \times 10^{-3}$ \\
\hline & 0.2 & 0.4038525842 & 0.4038290162 & $5.81 \times 10^{-3}$ & $2.36 \times 10^{-5}$ & $5.81 \times 10^{-3}$ \\
\hline & 0.3 & 0.4946163813 & 0.4945875165 & $7.12 \times 10^{-3}$ & $2.89 \times 10^{-5}$ & $7.12 \times 10^{-3}$ \\
\hline & 0.4 & 0.5711338018 & 0.5711004716 & $8.22 \times 10^{-3}$ & $3.33 \times 10^{-5}$ & $8.22 \times 10^{-3}$ \\
\hline & 0.5 & 0.6385470025 & 0.6385097382 & $9.19 \times 10^{-3}$ & $3.73 \times 10^{-5}$ & $9.19 \times 10^{-3}$ \\
\hline \multirow{5}{*}{0.5} & 0.1 & 0.3686652837 & 0.3686437692 & $5.30 \times 10^{-3}$ & $2.15 \times 10^{-5}$ & $5.30 \times 10^{-3}$ \\
\hline & 0.2 & 0.5213714443 & 0.5213410181 & $7.50 \times 10^{-3}$ & $3.04 \times 10^{-5}$ & $7.50 \times 10^{-3}$ \\
\hline & 0.3 & 0.6385470025 & 0.6385097382 & $9.19 \times 10^{-3}$ & $3.73 \times 10^{-5}$ & $9.19 \times 10^{-3}$ \\
\hline & 0.4 & 0.7373305676 & 0.7372875385 & $1.06 \times 10^{-2}$ & $4.30 \times 10^{-5}$ & $1.06 \times 10^{-2}$ \\
\hline & 0.5 & 0.8243606355 & 0.8243125275 & $1.19 \times 10^{-2}$ & $4.81 \times 10^{-5}$ & $1.19 \times 10^{-2}$ \\
\hline \multirow{5}{*}{1} & 0.1 & 0.5213714443 & 0.5213410181 & $7.50 \times 10^{-3}$ & $3.04 \times 10^{-5}$ & $7.50 \times 10^{-3}$ \\
\hline & 0.2 & 0.7373305676 & 0.7372875385 & $1.06 \times 10^{-2}$ & $4.30 \times 10^{-5}$ & $1.06 \times 10^{-2}$ \\
\hline & 0.3 & 0.9030418312 & 0.9029891315 & $1.30 \times 10^{-2}$ & $5.27 \times 10^{-5}$ & $1.30 \times 10^{-2}$ \\
\hline & 0.4 & 1.0427428890 & 1.0426820360 & $1.50 \times 10^{-2}$ & $6.09 \times 10^{-5}$ & $1.50 \times 10^{-2}$ \\
\hline & 0.5 & 1.1658219910 & 1.1657539560 & $1.68 \times 10^{-2}$ & $6.80 \times 10^{-5}$ & $1.68 \times 10^{-2}$ \\
\hline
\end{tabular}

coefficients $k_{1}$ and $k_{2}$, respectively, by using the least-squares method.

Figure 6 shows the influence of different $\alpha$ on the approximate solutions when $t=1, h=0.5,0 \leq x \leq 1,0 \leq y \leq 1$. Figure 6(a) presents approximate solutions for $\alpha=0.3$, Figure 6(b) presents approximate solutions for $\alpha=0.5$, Figure 6(c) presents approximate solutions for $\alpha=0.7$, and Figure 6(d) presents approximate solutions for $\alpha=0.9$.

From Figure 6, the larger the value of alfa, the smoother the image and the closer the image to the exact solution.

Using the least-squares residual power series method, the linearly independent functions can be verified when $\alpha=0.3,0.6$. The unknown coefficients $k_{1}$ and $k_{2}$ can be obtained by using the least-squares method. Table 6 shows the approximate solutions and the values of $|\widetilde{\operatorname{Res}}(x, t)|$ when $t=0.1, h=0.3, i=2$.

\section{Conclusion}

In this paper, we discuss the approximate solutions of the least-squares residual power series method. This method is an improvement on the classic residual power series method. We combine the least-squares method and residual power series method. We obtain more accurate approximate solutions with fewer expansion terms. The approximate solutions are presented by data and graphics. The results show that the approximate solutions solved by this method have 


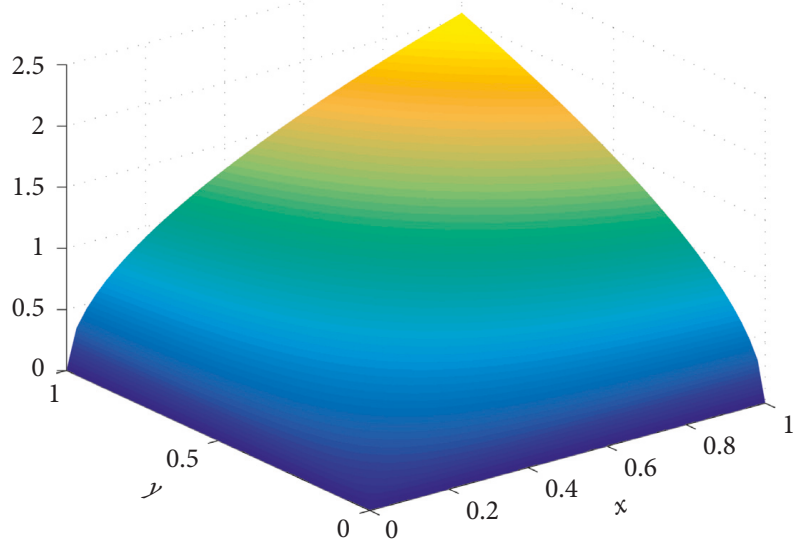

(a)

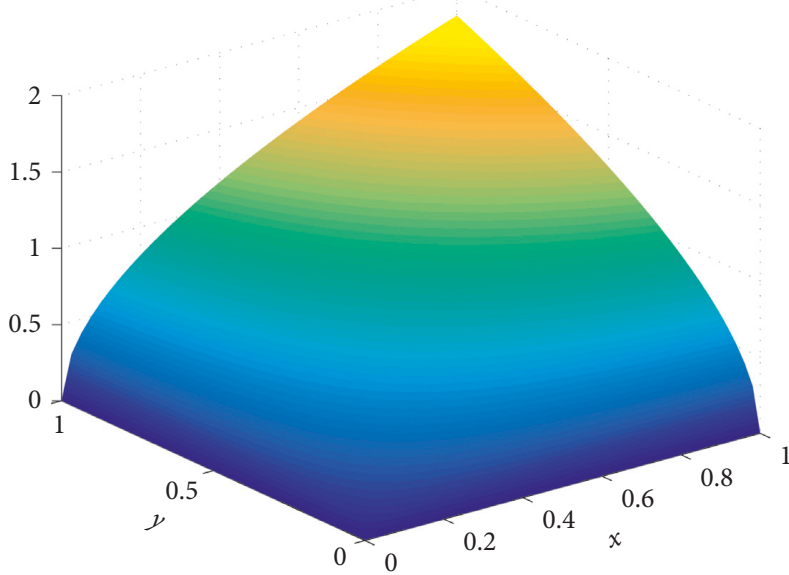

(c)

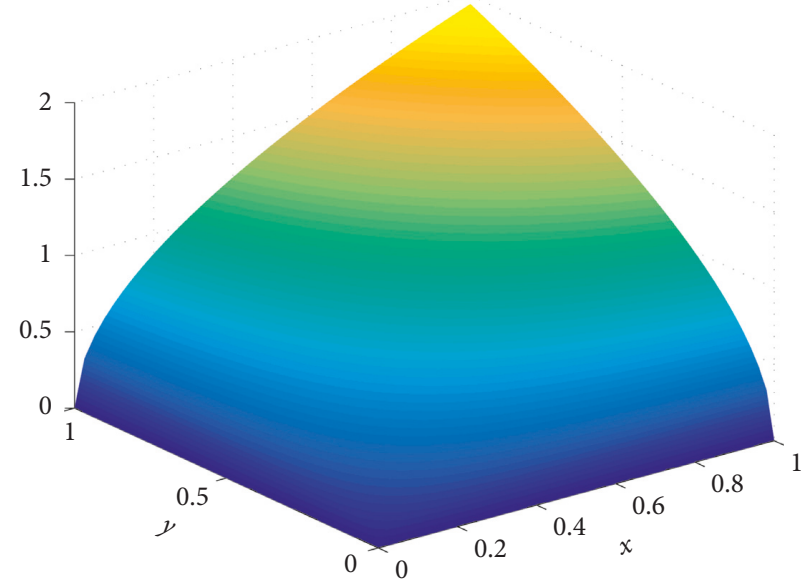

(b)

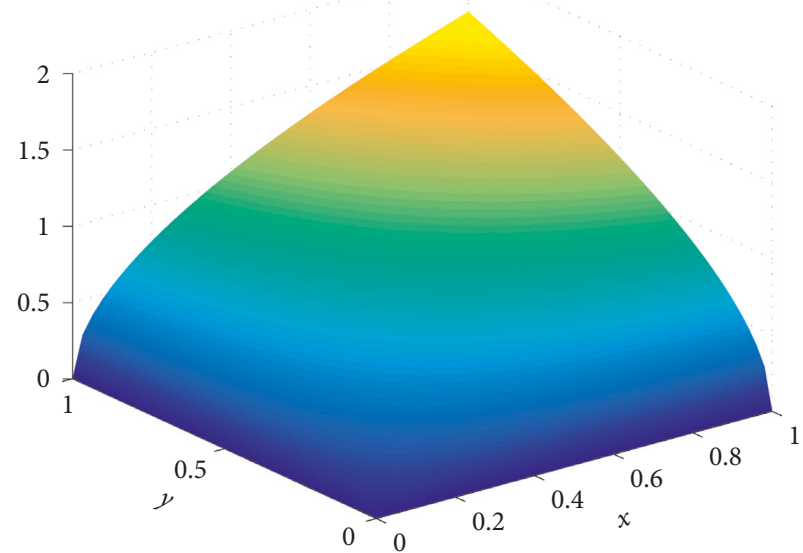

(d)

FIgURE 6: Approximate solution: (a) $\widetilde{u}(x, t, \alpha=0.3)$; (b) $\widetilde{u}(x, t, \alpha=0.5)$; (c) $\widetilde{u}(x, t, \alpha=0.7)$; (d) $\widetilde{u}(x, t, \alpha=0.9)$.

TABLE 6: The values of $|\widetilde{\operatorname{Res}}(x, t)|$ by LSRPSM, RPSM, and HPM for $\alpha=0.3,0.6$ and $t=0.1$.

\begin{tabular}{|c|c|c|c|c|c|c|c|c|c|}
\hline \multirow{2}{*}{$x$} & \multirow{2}{*}{$y$} & \multicolumn{4}{|c|}{$\alpha=0.3$} & \multicolumn{4}{|c|}{$\alpha=0.6$} \\
\hline & & $\tilde{u}$ & $|\widetilde{\operatorname{Res}}|_{\text {LSRPSM }}$ & $|\widetilde{\operatorname{Res}}|_{\text {RPSM }}$ & $|\operatorname{Res}|_{\mathrm{HPM}}$ & $\tilde{u}$ & $|\widetilde{\operatorname{ReS}}|_{\text {LSRPSM }}$ & $|\operatorname{Res}|_{\text {RPSM }}$ & $|\operatorname{Res}|_{\mathrm{HPM}}$ \\
\hline \multirow{3}{*}{0.1} & 0.1 & 0.120 & $5.388 \times 10^{-5}$ & $7.590 \times 10^{-4}$ & $7.590 \times 10^{-4}$ & 0.109 & $9.892 \times 10^{-5}$ & $1.546 \times 10^{-4}$ & $1.546 \times 10^{-4}$ \\
\hline & 0.2 & 0.169 & $7.620 \times 10^{-5}$ & $1.073 \times 10^{-3}$ & $1.073 \times 10^{-3}$ & 0.154 & $1.399 \times 10^{-4}$ & $2.187 \times 10^{-4}$ & $2.187 \times 10^{-4}$ \\
\hline & 0.3 & 0.202 & $9.333 \times 10^{-5}$ & $1.315 \times 10^{-3}$ & $1.315 \times 10^{-3}$ & 0.189 & $1.713 \times 10^{-4}$ & $2.678 \times 10^{-4}$ & $2.678 \times 10^{-4}$ \\
\hline \multirow{3}{*}{0.2} & 0.1 & 0.169 & $7.620 \times 10^{-5}$ & $1.073 \times 10^{-3}$ & $1.073 \times 10^{-3}$ & 0.154 & $1.399 \times 10^{-4}$ & $2.187 \times 10^{-4}$ & $2.187 \times 10^{-4}$ \\
\hline & 0.2 & 0.239 & $1.078 \times 10^{-4}$ & $1.518 \times 10^{-3}$ & $1.518 \times 10^{-3}$ & 0.218 & $1.978 \times 10^{-4}$ & $3.092 \times 10^{-4}$ & $3.092 \times 10^{-4}$ \\
\hline & 0.3 & 0.293 & $1.320 \times 10^{-4}$ & $1.859 \times 10^{-3}$ & $1.859 \times 10^{-3}$ & 0.267 & $2.423 \times 10^{-4}$ & $3.787 \times 10^{-4}$ & $3.787 \times 10^{-4}$ \\
\hline \multirow{3}{*}{0.3} & 0.1 & 0.207 & $9.333 \times 10^{-5}$ & $1.315 \times 10^{-3}$ & $1.315 \times 10^{-3}$ & 0.189 & $1.713 \times 10^{-4}$ & $2.678 \times 10^{-4}$ & $2.678 \times 10^{-4}$ \\
\hline & 0.2 & 0.293 & $1.320 \times 10^{-4}$ & $1.859 \times 10^{-3}$ & $1.859 \times 10^{-3}$ & 0.267 & $2.423 \times 10^{-4}$ & $3.787 \times 10^{-4}$ & $3.787 \times 10^{-4}$ \\
\hline & 0.3 & 0.359 & $1.616 \times 10^{-4}$ & $2.277 \times 10^{-3}$ & $2.277 \times 10^{-3}$ & 0.327 & $2.968 \times 10^{-4}$ & $4.639 \times 10^{-4}$ & $4.639 \times 10^{-4}$ \\
\hline
\end{tabular}

little error. In summary, this new technique is effective and accurate in finding approximate solutions for the timefractional differential equations.

\section{Data Availability}

The data used to support the findings of this study are available from the corresponding author upon request.

\section{Conflicts of Interest}

The authors declare that they have no conflicts of interest.

\section{Acknowledgments}

This study was supported by the National Natural Science Foundation of China (grant nos. 11701446, 11601420, and 
11401469), Natural Science Foundation of Shaanxi Province (2018JM1055), New Star Team of Xian University of Posts and Telecommunications, and Construction of Special Funds for Key Disciplines in Shaanxi Universities.

\section{References}

[1] B. Prieto, M. Martinez-Iturralde, L. Fontan, and I. Eloseguiau, "Analytical calculation of the slot leakage inductance in fractional-slot concentrated-winding machines," IEEE Transactions on Industrial Electronics, vol. 62, no. 5, pp. 2742-2752, 2015.

[2] J. T. Machado, "Numerical calculation of the left and right fractional derivatives," Journal of Computational Physics, vol. 293, pp. 96-103, 2015.

[3] R. K. Pandey and H. K. Mishra, "Homotopy analysis Sumudu transform method for time-fractional third order dispersive partial differential equation," Advances in Computational Mathematics, vol. 43, no. 2, pp. 1-19, 2016.

[4] A. Bekir and Ö. Güner, "The $G^{\prime} / G$-expansion method using modified Riemann-Liouville derivative for some space-time fractional differential equations," Ain Shams Engineering Journal, vol. 5, no. 3, pp. 959-965, 2014.

[5] S. H. Hosseinnia, A. Ranjbar, and S. Momani, "Using an enhanced homotopy perturbation method in fractional differential equations via deforming the linear part," Computers \& Mathematics with Applications, vol. 56, no. 12, pp. 31383149, 2008.

[6] Y. Nawaz, "Variational iteration method and homotopy perturbation method for fourth-order fractional integrodifferential equations," Computers \& Mathematics with Applications, vol. 61, no. 8, pp. 2330-2341, 2011.

[7] M. Alquran, "Analytical solutions of fractional foam drainage equation by residual power series method," Mathematical Sciences, vol. 8, no. 4, pp. 153-160, 2014.

[8] L. Wang and X. Chen, "Approximate analytical solutions of time fractional Whitham-Broer-Kaup equations by a residual power series method," Entropy, vol. 17, no. 9, pp. 6519-6533, 2015.

[9] Z. Korpinar and M. Inc, "Numerical simulations for fractional variation of $(1+1)$-dimensional Biswas-Milovic equation," Optik, vol. 166, pp. 77-85, 2018.

[10] M. Alquran, H. M. Jaradat, and M. I. Syam, "Analytical solution of the time-fractional Phi-4 equation by using modified residual power series method," Nonlinear Dynamics, vol. 90, no. 4, pp. 2525-2529, 2017.

[11] I. Komashynska, M. Alsmadi, A. Alhabahbeh, and A. Ateiwi, "Analytical approximate solutions of systems of multi-pantograph delay differential equations using residual powerseries method," Australian Journal of Basic and Applied Science, vol. 8, no. 10, pp. 664-675, 2014.

[12] A. Kumar, S. Kumar, and M. Singh, "Residual power series method for fractional Sharma-Tasso-Olever equation," Communications in Numerical Analysis, vol. 2016, no. 1, pp. 1-10, 2016.

[13] Y. Zhang, A. Kumar, S. Kumar, D. Baleanu, and X.-J. Yang, "Residual power series method for time-fractional Schrödinger equations," Journal of Nonlinear Sciences and Applications, vol. 9, no. 11, pp. 5821-5829, 2016.

[14] E. Abuteen and A. Freihet, "Analytical and numerical solution for fractional gas dynamic equations using residual power series method," in Proceedings of International Conference on Fractional Differentiation and its Applications (ICFDA) 2018, Amman, Jordan, July 2018.
[15] M. I. Syam, "Analytical solution of the fractional initial emden-fowler equation using the fractional residual power series method," International Journal of Applied and Computational Mathematics, vol. 4, no. 4, p. 106, 2018.

[16] A. Kumar and S. Kumar, "Residual power series method for fractional Burger types equations," Nonlinear Engineering, vol. 5, no. 4, pp. 235-244, 2016.

[17] H. Tariq and G. Akram, "Residual power series method for solving time-space-fractional Benney-Lin equation arising in falling film problems," Journal of Applied Mathematics and Computing, vol. 55, no. 1-2, pp. 683-708, 2017.

[18] R. Kumar and R. Koundal, "Generalized least square homotopy perturbations for system of fractional partial differential equations," 2018, http://arxiv.org/abs/1805.06650.

[19] C. Bota and B. Căruntu, "Approximate analytical solutions of nonlinear differential equations using the Least Squares Homotopy Perturbation Method," Journal of Mathematical Analysis and Applications, vol. 448, no. 1, pp. 401-408, 2017.

[20] H. Thabet and S. Kendre, "Modified least squares homotopy perturbation method for solving fractional partial differential equations," Malaya Journal of Matematik, vol. 06, no. 02, pp. 420-427, 2018.

[21] K. S. Miller and B. Ross, An Introduction to the Fractional Calculus and Fractional Differential Equations, Wiley, Hoboken, NJ, USA, 1993.

[22] A. A. Kilbas, H. M. Srivastava, and J. J. Trujillo, Theory and Applications of Fractional Differential Equations, Elsevier, Amsterdam, Netherlands, 2006.

[23] R. Almeida and D. F. M. Torres, "Calculus of variations with fractional derivatives and fractional integrals," Applied Mathematics Letters, vol. 22, no. 12, pp. 1816-1820, 2009.

[24] R. Almeida and D. F. M. Torres, "Necessary and sufficient conditions for the fractional calculus of variations with Caputo derivatives," Communications in Nonlinear Science and Numerical Simulation, vol. 16, no. 3, pp. 1490-1500, 2012.

[25] O. P. Agrawal, "Fractional variational calculus in terms of Riesz fractional derivatives," Journal of Physics A: Mathematical and Theoretical, vol. 40, no. 24, pp. 6287-6303, 2007.

[26] A. El-Ajou, O. Arqub, Z. Zhour, and S. Momani, "New results on fractional power series: theories and applications," Entropy, vol. 15, no. 12, pp. 5305-5323, 2013.

[27] A. El-Ajou, O. A. Arqub, and S. Momani, "Approximate analytical solution of the nonlinear fractional KdV-Burgers equation: a new iterative algorithm," Journal of Computational Physics, vol. 293, pp. 81-95, 2014.

[28] S. Kumar, A. Kumar, and D. Baleanu, "Two analytical methods for time-fractional nonlinear coupled BoussinesqBurger's equations arise in propagation of shallow water waves," Nonlinear Dynamics, vol. 85, no. 2, pp. 699-715, 2016.

[29] J. Zhang and L. Yin, "Residual power series method for the time fractional Fornberg-Whitham equation," International Journal of Dynamical Systems and Differential Equations, In press, https://www.inderscience.com/info/ingeneral/ forthcoming.php?jcode=ijdsde.

[30] M. G. Sakar, F. Erdogan, and A. Yıldırım, "Variational iteration method for the time-fractional Fornberg-Whitham equation," Computers \& Mathematics with Applications, vol. 63, no. 9, pp. 1382-1388, 2012.

[31] J. Zhang, Z. Wei, L. Yong, and Y. Xiao, "Analytical solution for the time fractional BBM-burger equation by using modified residual power series method," Complexity, vol. 2018, Article ID 2891373, 11 pages, 2018.

[32] M. E. Soh, C. D. Enyi, O. S. Iyiola, and J. D. Audu, “Approximate analytical solutions of strongly nonlinear fractional 
BBM-Burger's equations with dissipative term," Applied Mathematical Sciences, vol. 8, no. 155, pp. 7715-7726, 2014.

[33] M. Audu, K. Al-Khaled, and J. Chattopadhyay, "Analytical solutions of fractional population diffusion model: residual power series," Nonlinear Studies, vol. 22, no. 1, pp. 31-39, 2015.

[34] Y. Liu, Z. Li, and Y. Zhang, "Homotopy perturbation method to fractional biological population equation," Fractional Differential Calculus, vol. 1, no. 1, pp. 117-124, 2011. 


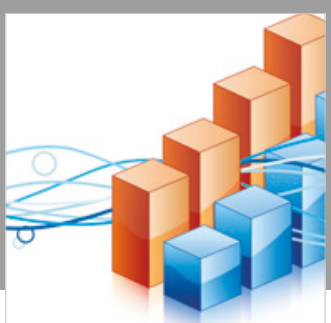

Advances in

Operations Research

\section{-n-m}
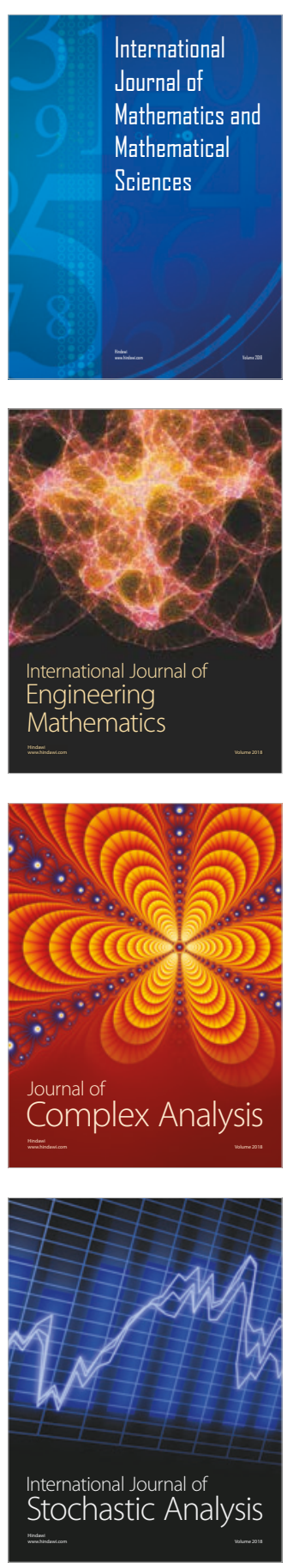
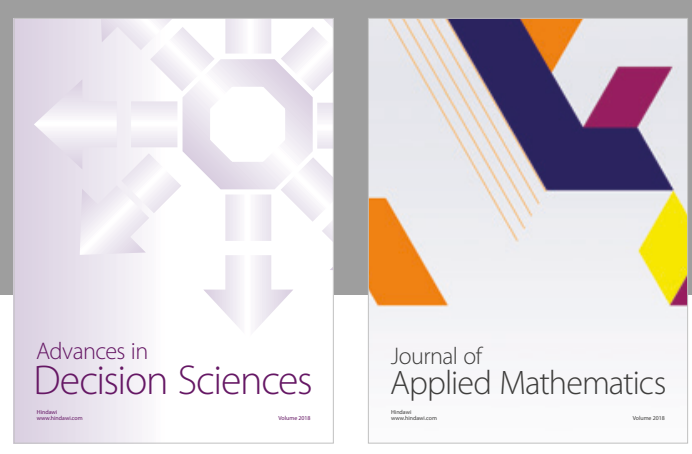

Journal of

Applied Mathematics
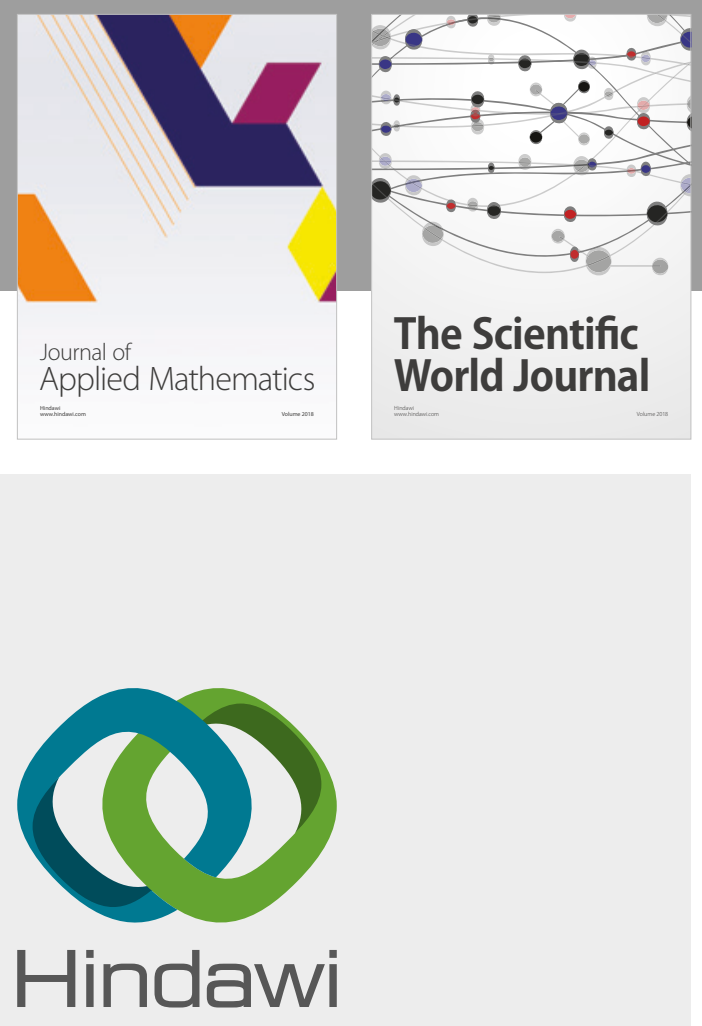

Submit your manuscripts at

www.hindawi.com

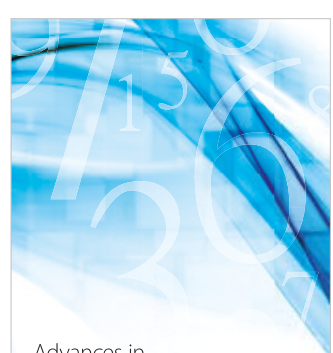

Advances in
Numerical Analysis
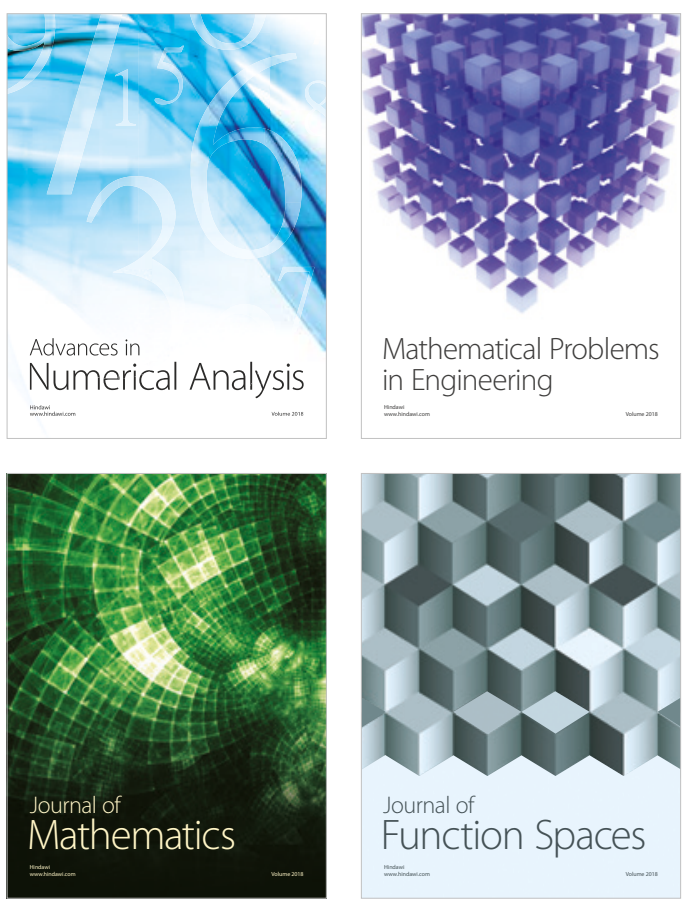

Mathematical Problems in Engineering

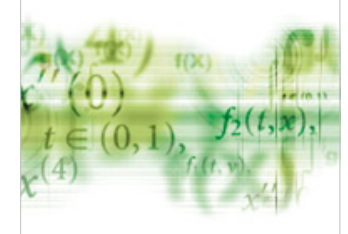

International Journal of

Differential Equations

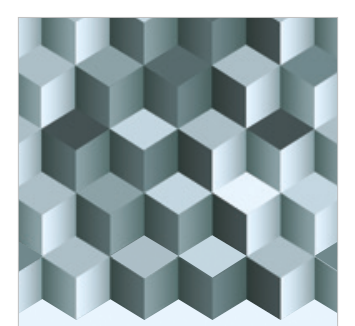

Journal of

Function Spaces
The Scientific

World Journal

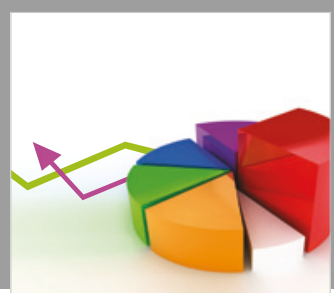

Journal of

Probability and Statistics
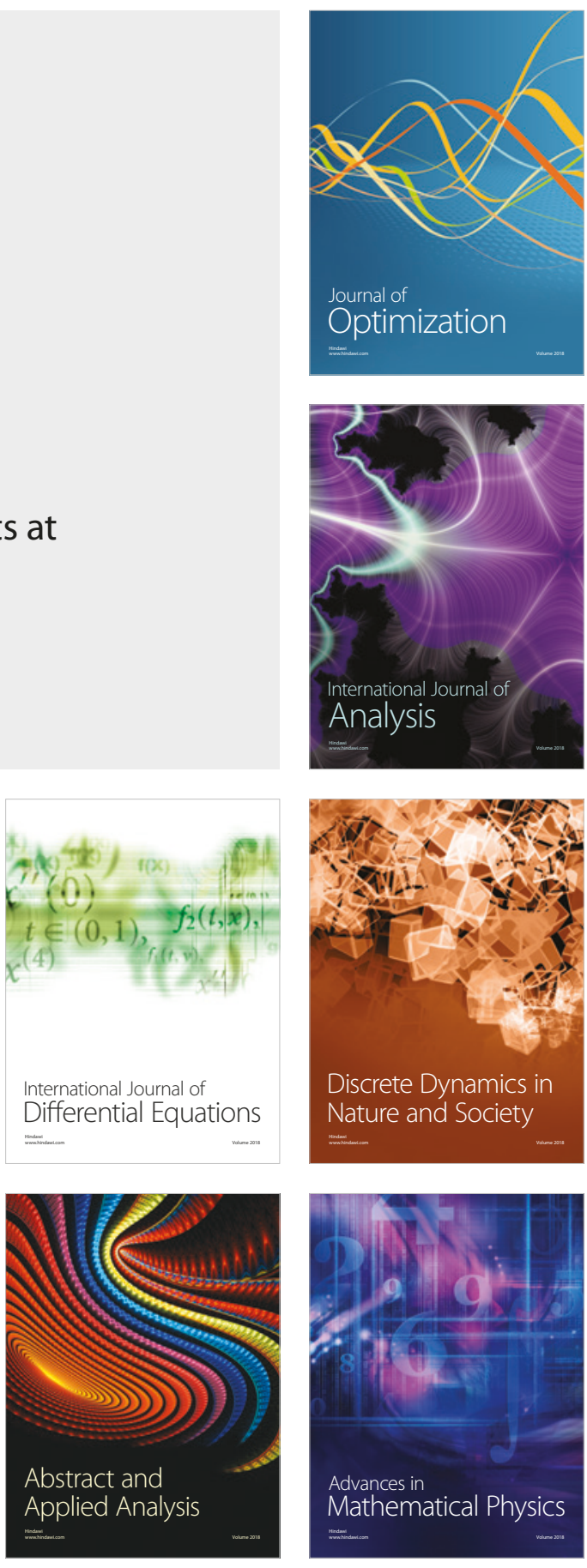